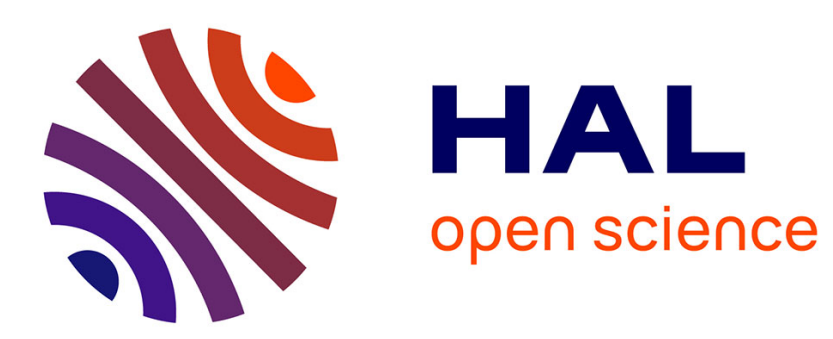

\title{
Efficient Computation of Dual Space and Directional Multiplicity of an Isolated Point
}

\author{
Angelos Mantzaflaris, Hamid Rahkooy, Zafeirakis Zafeirakopoulos
}

\section{To cite this version:}

Angelos Mantzaflaris, Hamid Rahkooy, Zafeirakis Zafeirakopoulos. Efficient Computation of Dual Space and Directional Multiplicity of an Isolated Point. Computer Aided Geometric Design, 2016, 47, pp.114-129. 10.1016/j.cagd.2016.05.002 . hal-01214195v2

\section{HAL Id: hal-01214195 \\ https://hal.inria.fr/hal-01214195v2}

Submitted on 11 May 2016

HAL is a multi-disciplinary open access archive for the deposit and dissemination of scientific research documents, whether they are published or not. The documents may come from teaching and research institutions in France or abroad, or from public or private research centers.
L'archive ouverte pluridisciplinaire HAL, est destinée au dépôt et à la diffusion de documents scientifiques de niveau recherche, publiés ou non, émanant des établissements d'enseignement et de recherche français ou étrangers, des laboratoires publics ou privés. 


\title{
Efficient Computation of Dual Space and Directional Multiplicity of an Isolated Point
}

\author{
Angelos Mantzaflaris ${ }^{\mathrm{a}}$, Hamid Rahkooy ${ }^{\mathrm{b}}$, Zafeirakis Zafeirakopoulos $^{\mathrm{c}}$ \\ ${ }^{a}$ Radon Institute for Computational and Applied Mathematics, Austrian Academy of \\ Sciences, Linz, Austria \\ ${ }^{b}$ RISC \& DK Computational Mathematics, Linz, Austria \\ ${ }^{c}$ Institute of Information Technologies, Gebze Technical University, Kocaeli, Turkey
}

\begin{abstract}
Isolated singularities typically occur at self-intersection points of planar algebraic curves, curve offsets, intersections between spatial curves and surfaces, and so on. The information characterizing the singularity can be captured in a local dual basis, expressing combinations of vanishing derivatives at the singular point. Macaulay's algorithm is a classic algorithm for computing such a basis, for a point in an algebraic set. The integration method of Mourrain constructs much smaller matrices than Macaulay's approach, by performing integration on previously computed elements.

In this work we are interested in the efficiency of dual basis computation, as well as its relation to orthogonal projection. First, we introduce an easy to implement criterion that avoids redundant computations during the computation of the dual basis, by deleting certain columns from the matrices in the integration method. In doing so, we explore general (non-monomial) bases for the associated primal quotient ring. Experiments show the efficient behaviour of the improved method. Second, we introduce the notion of directional multiplicity, which expresses the multiplicity structure with respect to an axis, and is useful in understanding the geometry behind projection. We use this notion to shed light on the gap between the degree of the generator of the elimination ideal and the corresponding factor in the resultant.
\end{abstract}

Keywords: multiplicity structure, dual space, directional multiplicity, Gröbner basis, algebraic curves

2000 MSC: $13 \mathrm{P} 10$, 2000 MSC: $68 \mathrm{~W} 30$

Email addresses: Angelos.Mantzaflaris@oeaw.ac.at (Angelos Mantzaflaris), rahkooy@risc.jku.at (Hamid Rahkooy), zafeirakopoulos@gtu.edu.tr (Zafeirakis Zafeirakopoulos)

Preprint submitted to Elsevier 


\section{Introduction}

Multiple points correspond to singular intersections between geometric objects. Therefore special care has to be taken in geometric algorithms in the vicinity of such points $[1,13]$. Singularities also arise while offsetting planar curves, and their robust computation is a long-standing problem. For example, in [22] the problem is treated using an algebraic approach. In implicit representations, one has access to evaluation of the level set function, rather than parameterized coordinates, therefore evaluation at arbitrary points in space is the basic tool at hand to be used for computations on this representation. For instance the topology or even the change of representation to implicit form is possible using solely the evaluation at arbitrary points $[2,6,8]$. Horner's scheme provides a stable and fast method to evaluate a polynomial in monomial representation. In the present paper we use value and derivative evaluations to identify isolated singular solutions via dual spaces.

The dual space of polynomial rings and algorithms for computing it, as well as the multiplicity structure of isolated points have been studied in different contexts in the literature, such as root deflation and certification, approximate root refinement, and so on $[9,12,18,19,20,21,23,31,35]$ and has been recently studied for the positive dimensional case in $[14,15]$. In the following we present a short literature review along with an introduction of notions, theorems and notation, which we will use in later sections.

Let $R=\mathbb{K}\left[x_{1}, \ldots, x_{n}\right]$ and consider it as a vector space over $\mathbb{K}$. Denote by $\hat{R}$ the dual of $R$. Let $\zeta=\left(\zeta_{1}, \ldots, \zeta_{n}\right) \in \mathbb{K}^{n}$ and $\mathbf{a}=\left(a_{1}, \ldots, a_{n}\right) \in \mathbb{N}^{n}$, and consider the differential operator $\partial_{\zeta}^{\mathbf{a}}$ that acts on $p \in R$ first by differentiation and then by evaluation at the point $\zeta$. In [28], Proposition 2.2 states that there is an isomorphism of $\mathbb{K}$-vector spaces between $\hat{R}$ and $\mathbb{K}\left[\left[\partial_{\zeta}\right]\right]$. This vector space, in general, is not finite dimensional. The orthogonal of an ideal $I$ of $R$, i.e., $I^{\perp}=\{\lambda \in \hat{R}: \lambda(f)=0 \quad \forall f \in I\}$ can be seen as a linear subspace of $\mathbb{K}\left[\left[\partial_{\zeta}\right]\right]$, for every $\zeta \in \mathbb{K}^{n}$, as shown in Proposition 2.6 of [28]. For the rest of this work, unless otherwise stated, we assume that $\zeta=0$ and we may omit the subscript, i.e., use $\partial^{\mathbf{a}}$ instead of $\partial_{\zeta}^{\mathbf{a}}$ and $\mathbb{K}[[\partial]]$ instead of $\mathbb{K}\left[\left[\partial_{\zeta}\right]\right]$.

In $[26,27]$, Marinari, Mora and Möller have shown that the $\mathfrak{m}_{\zeta}$-primary ideals are in one-to-one correspondence with the non-null finite-dimensional subspaces of $\mathbb{K}[\partial]$. Thus, the dual of an $\mathfrak{m}_{\zeta \text {-primary ideal can be treated computationally }}$ and its dimension is the multiplicity of the isolated point. From now on, given an ideal $I=\left\langle f_{1}, \ldots, f_{e}\right\rangle \subseteq R$, we denote by $Q_{\zeta}$, the $\mathfrak{m}_{\zeta}$-primary component of $I$ associated to an isolated point $\zeta$ in the variety of $I$. $D$ will stand for a basis for $Q_{\zeta}^{\perp}$. Therefore, according to Marinari, Mora and Möller $\langle D\rangle=Q_{\zeta}^{\perp}=I^{\perp} \cap \mathbb{K}\left[\partial_{\zeta}\right]$.

The maximum degree of the dual basis is also known as the depth. Together with the breadth, i.e. the co-rank of the Jacobian matrix, they are basic parameters that quantify the complexity of the multiplicity structure. The Nil-index of an $\mathfrak{m}_{\zeta}$-primary ideal $Q_{\zeta}$ is the maximum integer $\mathcal{N} \in \mathbb{N}$ such that $\mathfrak{m}_{\zeta}^{\mathcal{N}} \nsubseteq Q_{\zeta}$. There is a tight connection between the dual space of $\mathfrak{m}_{\zeta}$-primary ideals and their Nil-index given by Mourrain as Lemma 3.3 in [28], namely, considering the differential operators as polynomials, the maximum degree of the elements of 
$I^{\perp} \cap \mathbb{K}\left[\partial_{\zeta}\right]$ is equal to the Nil-index of $Q_{\zeta}$. Therefore, we can find the monomials of a basis $D$ for $Q^{\perp}$ by searching incrementally, i.e., degree by degree, among those monomials of $I^{\perp}$ that have degree at most the Nil-index.

For a recent review on computing the dual space see [24]. The dual space of polynomial rings and its properties and applications have been thoroughly reviewed in [9]. Concerning literature on computations related to the dual bases, $\mathrm{Wu}$ and Zhi worked on a symbolic-numeric method for computing the primary components and the differential operators [34], which is based on an algorithm for determining the dual space appearing in [31] by Stetter. A specialized algorithm for the case of breadth (Jacobian co-rank) one is devised in [17].

Below we present the main ideas behind the algorithms in the literature and in particular we explain Macaulay's algorithm [21] and the integration method $[23,28]$.

Due to the degree bound mentioned above, the algorithms that compute a basis $D$ for $Q^{\perp}$ proceed degree by degree. Let $D_{t}$ be the subset of $D$ that contains degree at most $t$ elements of $D$ (which is also a basis for the degree at most $t$ part of $Q^{\perp}$, denoted by $Q_{t}^{\perp}$ ). Obviously $D_{0}=\langle 1\rangle$. The algorithms extend $D_{t}$ into $D_{t+1}$, until $D_{t}=D_{t+1}$. Then we can conclude that $D=D_{t}$ and $t=\mathcal{N}$. We set $d_{i}:=d x_{i}$ for presentation reasons in what follows.

A historical work conducted by Macaulay [21] shows how to construct matrices in order to compute a basis for the dual space $Q^{\perp}$. It is based on a simple condition that the coefficients of the elements of the dual space must fulfill. Let $\Lambda=\sum_{|\alpha| \leq \mathcal{N}} \lambda_{\alpha} d^{\alpha}$, where we use the multi-index notation $d=d_{1} d_{2} \cdots d_{n}$. Then $\Lambda(f)=0$ for all $f \in I$ if and only if $\Lambda\left(x^{\beta} f_{i}\right)=0$ for all $\beta \in \mathbb{N}^{n}$ and $1 \leq i \leq e$. This observation, for $1 \leq|\beta| \leq \mathcal{N}$, reduces to checking that $\Lambda(f)=0$ for an infinite number of polynomials $f$ into checking the finitely many conditions that are given in the right hand side. Namely, it suffices to impose conditions on $\lambda_{\alpha}$ 's, the coefficients of $\Lambda$. For $1 \leq|\beta| \leq \mathcal{N}$, we obtain a system of linear homogeneous equations and construct the corresponding matrix. The rows of this matrix are labeled by $x^{\beta} f_{i}$ and the columns are labeled by $d^{\alpha}$. Every element in the kernel of this matrix is a coefficient vector, corresponding to an element of $Q_{\zeta}^{\perp}$. Therefore, the problem is now reduced into the kernel computation of some specific matrices.

This algorithm is still used widely, although it is not efficient. The main obstacles are the large size of the matrices and that the new matrices built contain as submatrices previously constructed matrices, repeating some computations. In [35] Zeng used the ideas in Stetter's algorithm [31] and introduced the closedness property in order to make Macaulay's matrices smaller.

Mourrain, in [28], gave a new algorithm based on integration, which constructs matrices of smaller size than those in Macaulay's algorithm. Assume that we have computed $D_{t-1}=\left\{\Lambda_{1}, \ldots, \Lambda_{m}\right\}$. The algorithm is based on integrating elements of $D_{t-1}$ in order to ansatz the elements of $D_{t}$ with symbolic 
coefficients $\lambda_{i j}$. More precisely,

$$
\Lambda=\sum_{i=1}^{m} \sum_{k=1}^{n} \lambda_{i k} \int_{k} \Lambda_{i}\left(d_{1}, \ldots, d_{k}, 0, \ldots, 0\right) .
$$

Then applying the following conditions on $\Lambda$ guarantees that $\Lambda \in Q_{t}^{\perp}$.

$$
\begin{aligned}
\forall 1 \leq k \leq e, \quad \Lambda\left(f_{k}\right) & =0 \\
\forall 1 \leq k<l \leq n, \quad \sum_{i=1}^{1} \lambda_{i k} d_{l}\left(\Lambda_{i}\right)-\sum_{i=1}^{1} \lambda_{i l} d_{k}\left(\Lambda_{i}\right) & =0 .
\end{aligned}
$$

From the above procedure one obtains a system of equations for $\lambda_{i j}$ to which a matrix $M_{t}$ is associated. Similar to Macaulay's algorithm, each vector in the kernel of $M_{t}$ determines the coefficients of an element in $Q_{t}^{\perp}$. The columns of $M_{t}$ are labeled by $\lambda_{i j} p_{i j}$ for $1 \leq i \leq e$ and $1 \leq j \leq n$, where $p_{i j}=$ $\int_{j} \Lambda_{i}\left(d_{1}, \ldots, d_{j}, 0, \ldots, 0\right)$. By abuse of notation and for simplifying the presentation, we sometimes use $\lambda_{i j}$ or $p_{i j}$ instead of $\lambda_{i j} p_{i j}$ in order to label the columns of $M_{t}$.

Although the integration method constructs much smaller matrices than Macaulay's algorithm, however, it might recompute $D_{t-1}$ in step $t$. In [23], the authors gave a method to overcome this problem. They use the tight connection between a dual basis for $Q_{t-1}^{\perp}$ and a primal basis for the degree $t-1$ part of the quotient $R / Q$. More precisely, if $D_{t-1}=\left\{\Lambda_{1}, \ldots, \Lambda_{m}\right\}$ is a basis of $Q_{t-1}^{\perp}$, where $\Lambda_{i}=d^{\beta_{i}}+\sum_{j=1}^{s-\mu} \lambda_{i j} d^{\gamma_{j}}$, then $\left\{x^{\beta_{1}}, \ldots, x^{\beta_{\mu}}\right\}$ is a basis for the degree $t-1$ part of $R / Q$. Details of the above has been explained in [28] and [23]. The following theorem by the first author and Mourrain results in avoiding redundant computations.

Theorem 1 (Lemma 3.4 in [23]). Let $D_{t}=\left\{\Lambda_{1}, \ldots, \Lambda_{m}\right\}$ be a basis of $Q_{t-1}^{\perp}$, such that it yields the basis $\left\{x^{\beta_{1}}, \ldots, x^{\beta_{\mu}}\right\}$ for the degree $t-1$ part of $R / Q$. An element $\Lambda \in \mathbb{K}[\partial]$ is not zero in $\Lambda \in D_{t} \backslash D_{t-1}$ if and only if in addition to (2) and (3), we impose

$$
\Lambda\left(x^{\beta_{i}}\right)=0, \quad \forall 1 \leq i \leq m .
$$

Equation (4) adds new rows to the matrix $M_{t}$, which results in a larger matrix that we denote by $\widetilde{M}_{t}$. However, by clever choice of a primal-dual basis, adding new rows can lead to removing some columns.

Example 1. Let

$$
\begin{aligned}
& f_{1}=x^{2}+(y-1)^{2}-1 \\
& f_{2}=y^{2} .
\end{aligned}
$$

Then for the root $(0,0)$, we have 


$$
\begin{gathered}
\text { Macaulay } \\
M_{1}=\begin{array}{ccc}
f_{1} \\
f_{2}
\end{array}\left(\begin{array}{ccc}
0 & 0 & -2 \\
0 & 0 & 0
\end{array}\right),
\end{gathered} \quad M_{1}=\begin{array}{r}
f_{1}\left(\begin{array}{ccc}
0 & 0 & -2 \\
f_{2} & d_{1} & d_{2} \\
0 & 0 & 0
\end{array}\right) .
\end{array}
$$

The kernel of $M_{1}$ yields the basis $D_{1}=\left\{1, d_{1}\right\}$. In the second step, we have

$$
\begin{aligned}
& \text { Macaulay }
\end{aligned}
$$

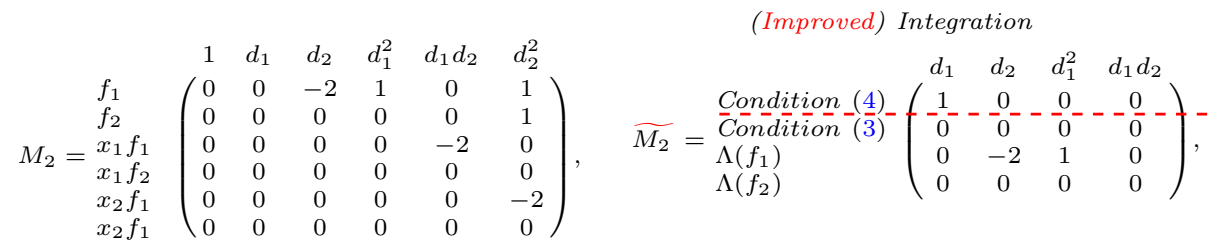

from which we obtain $D_{2}=\left\{1, d_{1}, 2 d_{1}^{2}+d_{2}\right\}$. Condition (4) implies that $\lambda_{1}=0$. Therefore we can remove from the integration method matrix the first column to obtain $\widetilde{M}_{2}$.

The algorithm runs until step 4 , during which we have $D_{3}=D_{4}=\left\{1, d_{1}, 2 d_{1}^{2}+\right.$ $\left.d_{2}, 2 d_{1}^{3}+d_{1} d_{2}\right\}$.

A detailed review of the integration method and its application to root deflation methods is given in [24]. In [3], Bates, Peterson and Sommese study the multiplicity of the primary components. Examining the multiplicity structure via deflation is exhibited in the work of Dayton and Zeng [7] and Leykin and Verschelde [16].

The present work was motivated (see [30]) and is closely connected to elimination theory. Marinari, Mora and Möller's work [26, 27] includes studying the behaviour of the dual space under projection, which is the base of our result related to the use of dual elements to study the elimination ideal in Section 3. There are different viewpoints on multiplicity structure. It can be studied via

- Gröbner bases: obtain global information on the elimination ideal and the multiplicity $[4,5]$.

- Resultants: obtain the multiplicity at a point, but not the elimination ideal $[10,11,32,33]$.

- Dual spaces: obtain the local structure of the multiplicity at that point as well as the elimination ideal and the Gröbner basis.

Apart from the application of Gröbner bases in computing a basis for the quotient ring and therefore the multiplicity, we will extensively use its elimination property [5] that allows computing elimination ideals. Mourrain in [28] proved that having a basis for the orthogonal of an ideal with an $\mathfrak{m}_{\zeta \text {-primary }}$ component, one can obtain the reduced Gröbner basis of this component.

Contributions. One contribution is improving the dual basis computation. Since the size of the matrices constructed in each step of the algorithms is the main 
obstacle in computations, we propose criteria that allow deleting some columns from the matrices in order to reduce the size of the matrices. Apart from those criteria, we introduce the notion of directional multiplicity, which gives more information about the singularity and its projections. An interesting interplay between the directional multiplicity and the degree of the elimination ideal is illustrated using intersections of algebraic curves.

For the integration method, the state of the art algorithm, in Proposition 5 we give an explicit generalization of the improvement in [23], as we detect and use a polynomial basis for the quotient rather than the monomial basis. The new primal bases are in accordance with [28, Prop. 3.7], which can be generalized for the case in question. Corollary 2 shows our criterion for deleting some columns such that the kernel of the new matrix only detects new members of a basis of the dual space, which avoids recomputing the lower degree basis elements that are obtained in the previous steps. The reduction of lower-degree elements has been employed in [23] using a different criterion; however, under certain circumstances this criterion can increase the number of rows of the matrix.

Outline. Section 2 includes our main results. The main improvements for computing the dual space are presented and their advantages are discussed. We introduce the notion of directional multiplicity and use the extended Buchberger diagram to illustrate it. We show bounds on the directional multiplicities with respect to Nil-index and the intersection multiplicity. Section 4 includes an empirical study of the algorithms presented. The theoretical expected behaviour is compared with the performance of the algorithms in practice. Finally, in Section 5 we summarize and conclude this work and provide future directions of research.

\section{Efficient Algorithms for Dual Basis}

Following the paradigm of [23], we provide modifications to the integration method and Macaulay's algorithms, which make computations more efficient. In particular, we give a more efficient criterion than (4) for the integration method.

Modifications on Integration Method. Recall that, in the integration method, the columns of $M_{t}$ are labeled by the $\lambda_{i j} p_{i j}$ 's appearing in $\Lambda$, but we may refer to the label just by $\lambda_{i j}$ or $p_{i j}$ for simplicity. Fix one of the $\lambda_{i j}$ 's and call it $\lambda$. We denote by $v_{\lambda}$ the column of $M_{t}$ that is indexed by $\lambda$ and $p_{\lambda}$ denotes the corresponding polynomial.

In step $t$ of the algorithm, there exists a correspondence between $D_{t}$ and $K_{t}$, a basis of $\operatorname{Ker}\left(M_{t}\right)$. If there exists a vector $q \in K_{t}$ for which the coordinate corresponding to $\lambda$ in this vector is nonzero, then we say that $v_{\lambda}$ is active in $D_{t}$. In case we explicitly know such a vector $q$, i.e., a particular element of the kernel corresponding to an element $E$ of $D_{t}$, then we say that $v_{\lambda}$ is active in $E$. Since $M_{t-1}$ is a sub-matrix of $M_{t}$, if it is clear from the context, by a column of $M_{t-1}$ we will refer to the corresponding column in $M_{t}$ as well. If $\lambda_{1}, \ldots, \lambda_{m}$

are the labels of some columns in $M_{t}$, then $M_{t}^{\lambda_{1}, \ldots, \lambda_{m}}$ denotes the submatrix of 
$M_{t}$ that is obtained by deleting columns $\lambda_{1}, \ldots, \lambda_{m}$ from $M_{t}$. We work on $M_{t}$ rather than $\widetilde{M}_{t}$ in this section, although many of our arguments hold for $\widetilde{M}_{t}$ as well.

We start with a proposition that provides us with an improvement on the integration method, related to (4).

Proposition 1. Let $M_{t}, M_{t-1}, D_{t}, \Lambda_{i}(1 \leq i \leq m), \lambda, p_{\lambda}$ and $v_{\lambda}$ be as above. Then the following hold.

1. If $v_{\lambda}$ is a column of $M_{t}$, then $v_{\lambda}$ is active in $D_{t}$ if and only if $v_{\lambda}$ can be reduced to zero by other columns of $M_{t}$.

2. For all $1 \leq i \leq m$, if $v_{\lambda_{i}}$ is active in $\Lambda_{i}, K_{t}^{\lambda_{i}}$ is a basis for $\operatorname{Ker}\left(M_{t}^{\lambda_{i}}\right)$ and $D_{t}^{\lambda_{i}}$ is the set of its corresponding dual elements, then $\left\{\Lambda_{i}\right\} \cup D_{t}^{\lambda_{i}}$ is a basis for the degree $t$ part of $Q^{\perp}$. Moreover, if $v_{\lambda_{i}}$ is active in $\Lambda_{i}$, but is not active in $\Lambda_{j}$ for $1 \leq j \neq i \leq m$, then there exists a basis $D_{t}^{\lambda_{i}}$ such that $\Lambda_{j} \in D_{t}^{\lambda_{i}}, j \neq i$.

3. Let $K_{t}^{\lambda_{1} \ldots \lambda_{m}}$ be a basis for $\operatorname{Ker}\left(M_{t}^{\lambda_{1} \ldots \lambda_{m}}\right)$ and $D_{t}^{\lambda_{1} \ldots \lambda_{m}}$ be the set of its corresponding dual elements. If for all $1 \leq i \leq m, v_{\lambda_{i}}$ is active in $\Lambda_{i}$ but not active in $\Lambda_{1}, \ldots, \Lambda_{i-1}$, then $D_{t-1} \cup D_{t}^{\lambda_{1} \ldots \lambda_{m}}$ is a basis for the degree $t$ part of $Q^{\perp}$.

Proof. 1. Let $v_{\lambda}, v_{1}, \ldots, v_{k}$ denote the columns of $M_{t}$ and $p_{\lambda}, p_{1}, \ldots, p_{k}$ be the polynomials labeling the columns of $M_{t}$. Then $v_{\lambda}$ can be reduced to zero by $v_{1}, \ldots, v_{k}$ if and only if there exist $c_{1}, \ldots, c_{k} \in \mathbb{K}$, such that $v_{\lambda}=c_{1} v_{1}+\cdots+c_{k} v_{k}$, or equivalently $v_{\lambda}-c_{1} v_{1}-\cdots-c_{k} v_{k}=0$. This holds if and only if $q:=$ $\left(1, c_{1}, \cdots, c_{k}\right) \in K_{t}$, which holds if and only if $\Lambda^{\prime}:=p_{\lambda}-c_{1} p_{1}-\cdots-c_{k} p_{k} \in D_{t}$ (note that this is exactly the fact that $\Lambda^{\prime}$ in $D_{t}$ corresponds to $q \in K_{t}$ ) The latter is the case if and only if $v_{\lambda}$ is active in $\Lambda^{\prime}$, or equivalently $v_{\lambda}$ is active in $D_{t}$.

2. Fix $1 \leq i \neq j \leq m$ and let $q_{i}$ and $q_{j}$ be the elements of $K_{t}$ corresponding to $\Lambda_{i}$ and $\Lambda_{j}$ in $D_{t}$, respectively.

First we prove that for all $\Lambda^{\prime} \in D_{t}$ if $\Lambda^{\prime} \neq \Lambda_{i}$, then $\Lambda^{\prime} \in\left\langle D_{t}^{\lambda_{i}} \cup\left\{\Lambda_{i}\right\}\right\rangle$. Let $q^{\prime}$ be the corresponding element of $\Lambda^{\prime}$ in $K_{t}$. If $v_{\lambda_{i}}$ is not active in $\Lambda^{\prime}$, then by part 1 it cannot be reduced to zero by the active columns in $\Lambda^{\prime}$. So the column $v_{\lambda_{i}}$ is not involved in computing $\Lambda^{\prime}$ via column reducing in $M_{t}$. Thus, $\Lambda^{\prime}$ can be computed via column reducing in $M_{t}^{\lambda_{i}}$. Let $q^{\prime}$ be the corresponding element to $\Lambda^{\prime}$ in $\operatorname{Ker}\left(M_{t}\right)$. Then $q^{\prime} \in \operatorname{Ker}\left(M_{t}^{\lambda_{i}}\right)$. This means that $\Lambda^{\prime} \in\left\langle D_{t}^{\lambda_{i}}\right\rangle$.

If $v_{\lambda_{i}}$ is active in $\Lambda^{\prime}$, then we prove that there exists a $\Lambda^{\prime \prime}$ in $D_{t}^{\lambda_{i}}$ such that $\Lambda^{\prime}=\Lambda_{i}+\Lambda^{\prime \prime}$. Let $q^{\prime} \in K_{t}$ be the element corresponding to $\Lambda^{\prime} \in D_{t}$, such that that the first coordinate of $q^{\prime}$ corresponds to $v_{\lambda_{i}}$. Take $q^{\prime}=\left(1, b_{1}, \ldots, b_{k}\right)$. Then we have that $v_{\lambda_{i}}+b_{1} v_{1}+b_{2} v_{2}+\cdots b_{k} v_{k}=0$, where the columns $v_{1}, \ldots, v_{k}$ are as in the proof of part 1. Also, again as in the proof of the part $1, v_{\lambda_{i}}=$ $c_{1} v_{1}+\cdots+c_{k} v_{k}$. Therefore $\left(b_{1}-c_{1}\right) v_{1}+\cdots+\left(b_{k}-c_{k}\right) v_{k}=0$, which means that $\left(0, b_{1}-c_{1}, \ldots, b_{k}-c_{k}\right) \in \operatorname{Ker}\left(M_{t}\right)$, and therefore $q^{\prime \prime}:=\left(b_{1}-c_{1}, \ldots, b_{k}-c_{k}\right) \in$ $\operatorname{Ker}\left(M_{t}^{\lambda}\right)$. So one can construct a basis $K_{t}^{\lambda_{i}}$ in such a way that $q^{\prime \prime} \in K_{t}^{\lambda_{i}}$. Let $\Lambda^{\prime \prime}$ be the member of $D_{t}^{\lambda_{i}}$ corresponding to $q^{\prime \prime}$. Then $\Lambda^{\prime}=\Lambda_{i}+\Lambda^{\prime \prime}$. 
Secondly we note that if $v_{\lambda_{i}}$ is not active in $\Lambda_{j}$, for $1 \leq j \neq i \leq m$, then by the above argument, one can compute a basis $K_{t}^{\lambda_{i}}$ (and respectively, $D_{t}^{\lambda_{i}}$ ) in such a way that $\Lambda_{j} \in D_{t}^{\lambda_{i}}$.

Thus, every element of $D_{t}$ can be obtained from $\Lambda_{i}$ and an element of $K_{t}^{\lambda_{i}}$ and therefore $\left\langle D_{t}\right\rangle \subseteq\left\langle\left\{\Lambda_{i}\right\} \cup D_{t}^{\lambda_{i}}\right\rangle$. Since the elements of $\left\{\Lambda_{i}\right\} \cup D_{t}^{\lambda_{i}}$ are linearly independent, we have $\left\langle\left\{\Lambda_{i}\right\} \cup D_{t}^{\lambda_{i}}\right\rangle=\left\langle D_{t}\right\rangle=Q^{\perp}$.

3. Let $K_{t}^{v_{\lambda_{1}} \cdots v_{\lambda_{m}}}$ be a basis for $\operatorname{Ker}\left(M_{t}^{\lambda_{1} \cdots \lambda_{m}}\right)$ and $D_{t}^{\lambda_{1} \cdots \lambda_{m}}$ the corresponding dual elements. As in the proof of the previous parts, let $K_{t}$ be a basis for $\operatorname{Ker}\left(M_{t}\right)$ and $q_{1}, \ldots, q_{m} \in K_{t}$ correspond to $\Lambda_{1}, \ldots, \Lambda_{m}$ respectively. Then from the proof of part 2 we have that $\left\{q_{1}\right\} \cup K_{t}^{\lambda_{1}}$ is a basis for $\operatorname{Ker}\left(M_{t}\right)$. Also by part 2 of the proposition, $q_{2}, \ldots, q_{m} \in\left\langle K_{t}\right\rangle$ and correspondingly $\Lambda_{2}, \ldots, \Lambda_{m} \in\left\langle D_{t}^{\lambda_{1}}\right\rangle$. Now consider the matrix $M_{t}^{\lambda_{1}}$ and the basis $D_{t}^{\lambda_{1}}$ obtained from it. Since $v_{\lambda_{2}}$ is active in $\Lambda_{2}$ (which corresponds to $q_{2}$ in $K_{t}$ ), and it is not active in $\Lambda_{1}$, then we can apply part 2 of the proposition to the matrix $M_{t}^{\lambda_{1}}$ and the basis $D_{t}^{\lambda_{1}}$ obtained by it. Then we will have that $\left\{q_{2}\right\} \cup K_{t}^{\lambda_{1} \lambda_{2}}$ is a basis for $\operatorname{Ker}\left(M_{t}^{\lambda_{1}}\right)$ and $q_{3}, \ldots, q_{m} \in\left\langle K_{t}^{\lambda_{1} \lambda_{2}}\right\rangle$. Correspondingly, $\Lambda_{3}, \ldots, \Lambda_{m} \in\left\langle D_{t}^{\lambda_{1} \lambda_{2}}\right\rangle$. This implies that $\left\{q_{1}, q_{2}\right\} \cup K_{t}^{\lambda_{1} \lambda_{2}}$ is a basis for $\operatorname{Ker}\left(M_{t}\right)$. Continuing with $v_{\lambda_{i}}$, for $i \geq 3$, and considering the assumption that $v_{\lambda_{i}}$ is not active in $\Lambda_{1}, \ldots, \Lambda_{i-1}, j \neq i$, we finally get $\left\{q_{1}, \ldots, q_{m}\right\} \cup K_{t}^{\lambda_{1} \ldots \lambda_{m}}$ as a basis for $\operatorname{Ker}\left(M_{t}\right)$ and correspondingly $\left\{\Lambda_{1}, \ldots, \Lambda_{m}\right\} \cup D_{t}^{\lambda_{1} \ldots \lambda_{m}}$ as a basis for the degree $t$ part of $Q^{\perp}$.

The above proposition shows that deleting specific columns from $M_{t}$ avoids re-computing the basis elements of degree at most $t-1$, which were already computed in the previous steps. Not every set of $m$ active columns will give degree $t$ elements of a basis. In fact, if we delete two columns that are both active in two different basis members of $D_{t-1}$, then we may not obtain some members of $D_{t}$. For instance, let $D_{2}=\left\{\Lambda_{1}=d_{1}+d_{2}+d_{1}^{2}+d_{2}^{2}, \Lambda_{2}=d_{1}+d_{2}+2 d_{1}^{2}+d_{1} d_{2}\right\}$ and $\Lambda^{\prime}=d_{1}+d_{2}^{3} \in \operatorname{Ker}\left(M_{3}\right)$. Then $\Lambda^{\prime} \notin \operatorname{Ker}\left(M_{3}^{d_{1} d_{2}}\right)$.

In the following we show how to detect columns $v_{\lambda_{1}}, \ldots, v_{\lambda_{m}}$ that satisfy the assumption of part 3 of Proposition 1. This is done via changing the basis $\left\{\Lambda_{1}, \ldots, \Lambda_{m}\right\}$ into a new reduced basis $\left\{\Lambda_{1}^{\prime}, \ldots, \Lambda_{m}^{\prime}\right\}$, in which the leading terms satisfy the assumptions of part 3 of Proposition 1.

Let $D_{t-1}=\left\{\Lambda_{1}, \ldots, \Lambda_{m}\right\}$ as above. In the improvement of the integration method given in [23], having $D_{t-1}$, one can construct a matrix $D^{\prime}$ in order to obtain a basis for the degree $t$ part of $R / Q$, so that (4) can be applied (Equation (7) in [23]). Below we show constructing a similar, but smaller matrix which gives the desired set of active columns. Same as $M_{t}$, the columns of this matrix are labeled by the coefficients/polynomials that appear in $\Lambda$ in 1. Also same as $D^{\prime}$, the rows of this matrix come from $\Lambda_{1}, \ldots, \Lambda_{m}$. Let $v_{\lambda_{1}}, \ldots, v_{\lambda_{u}}$ be the columns of $M_{t}$ such that they are active in $D_{t-1}$. Construct the following matrix 
containing the columns $v_{\lambda_{1}}, \ldots, v_{\lambda_{u}}$

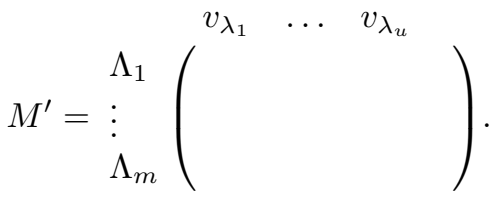

Changing $M^{\prime}$ into a row echelon form matrix, after moving the pivot columns to the left hand, we arrive at a matrix of the form

$$
G^{\prime} M^{\prime} P^{\prime}=\begin{gathered}
\Lambda_{1}^{\prime} \\
\Lambda_{2}^{\prime} \\
\vdots \\
\Lambda_{m}^{\prime}
\end{gathered}
$$

where diagonal entries are nonzero and $G^{\prime}$ is the matrix that takes care of the operations done for the row echelon form and $P^{\prime}$ is the matrix that takes care of the permutation of the columns. Note that the diagonal entries of $G^{\prime} M^{\prime} P^{\prime}$ are non-zero and the matrix is upper diagonal. This is because otherwise, if we obtain a zero row in $G^{\prime} M^{\prime} P^{\prime}$, that row is linearly dependent to the other rows. But this is in contradiction with $\Lambda_{1}, \ldots, \Lambda_{m}$ (and therefore $\Lambda_{1}^{\prime}, \ldots, \Lambda_{m}^{\prime}$ as their linear combinations) being linearly independent. Then our basis will satisfy the conditions of part 3 of Proposition 1.

Now we are ready to prove the following, which provides us with an algorithmic improvement of the integration method, more efficient than (4).

\section{Corollary 2. (Criterion for Deleting Active Columns)}

Let $D_{t-1}=\left\{\Lambda_{1}, \cdots, \Lambda_{m}\right\}, M_{t}, D_{t}, v_{\lambda_{1}}, \ldots, v_{\lambda_{u}}$ and $G^{\prime} M^{\prime} P^{\prime}$ be as above and (by abuse of notation) let $v_{\lambda_{1}}, \ldots, v_{\lambda_{m}}$ be the columns of $M_{t}$ corresponding to the first $m$ columns in $G^{\prime} M^{\prime} P^{\prime}$. Also let $K_{t}^{\lambda_{1} \ldots \lambda_{m}}$ be a basis for $\operatorname{Ker}\left(M_{t}^{\lambda_{1} \ldots \lambda_{m}}\right)$ and $D_{t}^{\lambda_{1} \ldots \lambda_{m}}$ be the set of its corresponding dual elements. Then $D_{t-1} \cup D_{t}^{\lambda_{1} \ldots \lambda_{m}}$ is a basis for the degree $t$ part of $Q^{\perp}$.

Proof. We only need to prove that the columns $v_{\lambda_{1}}, \ldots, v_{\lambda_{m}}$ in $G^{\prime} M^{\prime} P^{\prime}$ satisfy the conditions of part 3 of Proposition 1 . This is the case because for all $1 \leq$ $i \leq m, v_{\lambda_{i}}$ has zero in coordinates $i+1, \ldots, m$ and has non-zero coordinate $i$, which is the row corresponding to $\Lambda_{i}$. This means that for all $1 \leq i \leq m, v_{\lambda_{i}}$ is not active in $\Lambda_{1}, \ldots, \Lambda_{i-1}$. The result follows directly from Proposition 1.

Corollary 2 provides us with an optimization in the integration method. Assume that the monomials $x^{\mathbf{a}_{1}}, \ldots, x^{\mathbf{a}_{m}}$ form a basis for the degree $t-1$ part of $R / Q$. If the monomial $d x^{\mathbf{a}_{i}}$ appears only once in $\Lambda$ in (1), then applying (4), we have that

$$
\Lambda\left(x^{\mathbf{a}_{i}}\right)=\lambda_{i} d x^{\mathbf{a}_{i}}\left(x^{\mathbf{a}_{i}}\right)=\lambda_{i}=0 .
$$


This gives us an equation which adds a row to $M_{t}$. However, instead of adding the corresponding row to $M_{t}$, one can just plug in $\lambda_{i}=0$ in the other equations obtained from (2) and (3). This will remove $\lambda_{i}$ from the other equations, or equivalently will remove the column $v_{\lambda_{i}}$ from $M_{t}$. If we let $v_{\lambda_{i}}$ be the only column of $M_{t}$ such that its label contains $d x^{\mathbf{a}_{i}}$, then $v_{\lambda_{i}}$ is active in $\Lambda_{i}$ and therefore according to Corollary 2, one can delete it from $M_{t}$ in order to avoid re-computing $D_{t-1}$.

For a dual polynomial $p \in \mathbb{K}[\partial]$ we define its primal to be the polynomial $\widehat{p} \in \mathbb{K}\left[x_{1}, \ldots, x_{n}\right]$ obtained by substituting formally $d x_{i}$ with $x_{i}$ in $p$. In the following we show how our construction may be used to obtain a basis for the quotient ring.

Proposition 3. Let $v_{\lambda_{1}}, \ldots, v_{\lambda_{m}}$ be the columns deleted by the criterion in Corollary 2. Also let $p_{1}, \ldots, p_{m} \in \mathbb{K}[\partial]$ be the dual polynomials corresponding to the coefficients $\lambda_{1}, \ldots, \lambda_{m}$ of $\Lambda$ in (1). Consider the primals $\widehat{p_{1}}, \ldots, \widehat{p_{m}} \in$ $\mathbb{K}\left[x_{1}, \ldots, x_{n}\right]$ of $p_{1}, \ldots, p_{m}$. Then there exist monomials $\left\{\widehat{l_{1}}, \ldots, \widehat{l_{m}}\right\}$ with $\widehat{l_{i}} \in$ $\operatorname{supp}\left(p_{i}\right)$ which form a basis for the degree $t-1$ part of $R / Q$.

Proof. Each column $v_{\lambda_{i}}$ in Corollary 2 implies that in the dual basis we have an element $\Lambda_{i}^{\prime}$ with $\Lambda_{i}^{\prime}\left(\widehat{p}_{i}\right)=c_{i} \neq 0$. These elements correspond to the rows of $G^{\prime} M^{\prime} P^{\prime}$ in (6). Therefore, for every $i=1, \ldots, m$ there exists a dual monomial $l_{i}$ such that $\widehat{l_{i}}$ is in the support of $p_{i}$. We can diagonalize $G^{\prime} M^{\prime} P^{\prime}$ and obtain dual elements with $\Lambda_{j}^{\prime}\left(\widehat{p}_{i}\right)=0$ for all $j \neq i$. Therefore the dual monomial $l_{i}$ does not appear in any other $\Lambda_{j}, j \neq i$. We deduce that $\left\{\widehat{l_{1}}, \ldots, \widehat{l_{m}}\right\}$ is a set of distinct monomials and $\Lambda_{i}\left(\widehat{l_{j}}\right)=\lambda_{i} \delta_{i j}$, where $\delta_{i j}$ is the Kronecker delta. Moreover, each $\widehat{l_{i}}$ is non-zero in $R / Q$, since it is not annihilated by the corresponding $\Lambda_{i}^{\prime}$. Therefore, these monomials are the basis of the degree $t-1$ part of $R / Q$ which is dual to $\left\{\Lambda_{1}, \ldots, \Lambda_{m}\right\}$, after scaling the coefficients $\lambda_{i}$ to 1 .

Proposition 3 implies that the criterion for deleting active columns can be viewed as adding the equation $\Lambda\left(\widehat{p}_{i}\right)=0$ for $1 \leq i \leq m$ to the integration method's conditions. Same as (4), this equation leads to adding rows to $M_{t}$, however those rows are of the form $(0, \ldots, 0, c, 0, \ldots, 0)$, where $c$ is a nonzero element in coordinate $i$ for $1 \leq i \leq m$ and therefore they result in deleting the corresponding columns. Moreover, one can obtain the normal form from the dual basis obtained by our construction, as we show in the following

Proposition 4. With the same notation as above, if the set $\left\{\widehat{p_{1}}, \ldots, \widehat{p_{m}}\right\} \subseteq$ $\mathbb{K}\left[x_{1}, \ldots, x_{n}\right]$ is a (not necessarily monomial) basis of $R / Q$, then it's dual basis is

$$
\Lambda_{i}=\frac{1}{\left\|p_{i}\right\|^{2}} p_{i}+\sum_{j=1}^{k} \lambda_{i j} m_{j} \quad, \quad 1 \leq i \leq m,
$$

where $m_{j} \notin Q$ are trailing monomials and $\|\cdot\|$ is the Euclidean norm of the coefficients. Therefore the normal form of any $g \in \mathbb{K}\left[x_{1}, \ldots, x_{n}\right]$ with respect to 
$Q$ and $\left\{\widehat{p_{1}}, \ldots, \widehat{p_{m}}\right\}$ is

$$
N F(g)=\sum_{i=1}^{m} \Lambda_{i}(g) \widehat{p_{i}}
$$

Proof. From the proof of Proposition 3, it is clear that $\left\{\widehat{p_{1}}, \ldots, \widehat{p_{m}}\right\}$ are linearly independent in $R / Q$. Observe that $p_{i}\left(\widehat{p}_{i}\right)=\left\|p_{i}\right\|^{2}$ therefore when scaling $\Lambda_{i}^{\prime}$ in (6) by this constant we obtain that $\Lambda_{i}\left(\widehat{p_{j}}\right)=\delta_{i j}$. The remainder of the proof is exactly the same as the proof of Lemma 2.4 in [23].

If $\left\{\widehat{p_{1}}, \ldots, \widehat{p_{m}}\right\} \subseteq \mathbb{K}\left[x_{1}, \ldots, x_{n}\right]$ is an arbitrary basis of the quotient $R / Q$ and $\left\{p_{1}, \ldots, p_{m}\right\} \subseteq \mathbb{K}[\partial]$ are the corresponding dual polynomials, then removing from each $\widehat{p}_{i}$ the monomials that are in $Q$, we will obtain a new basis for $R / Q$. Thus this assumption holds without loss of generality and we generalize Lemma 3.4 from [23].

Proposition 5. Let $\left\{\widehat{p_{1}}, \ldots, \widehat{p_{m}}\right\} \subseteq \mathbb{K}\left[x_{1}, \ldots, x_{n}\right]$ be a basis for the degree $t$ part of $R / Q$ such that no monomial of $p_{i}$ is in $Q$. A non-zero element $\Lambda \in \mathbb{K}[\partial]$ is not zero in $Q_{t}^{\perp} \backslash Q_{t-1}^{\perp}$ if and only if in addition to (2) and (3) it satisfies $\Lambda\left(\widehat{p_{i}}\right)=0$ for $1 \leq i \leq m$.

Constructing matrices $M^{\prime}$ and $G^{\prime} M^{\prime} P^{\prime}$ in order to choose particular active columns and deleting them is a special case of the above proposition. We have the following generalization of Proposition 3.7 in [28], which gives a Gröbner basis of the primary component corresponding to the isolated point in question having its dual basis.

Proposition 6. Let $\prec$ be a term order and $\widehat{m_{j}}, m_{j}, \widehat{p}_{i}, p_{i}, \Lambda_{i}, 1 \leq i \leq m, 1 \leq$ $j \leq k$ be as in Proposition 4. Also let $w_{1}, \ldots, w_{s}$ be the monomials different from lt $\left(\widehat{p}_{i}\right), i=1 \ldots, m$. Write $w_{i}=\sum_{j=1}^{m} \gamma_{i j} \widehat{p_{j}}$ and let

$$
W=\left\{g_{w_{i}}:=w_{i}+\sum_{j=1}^{m} \gamma_{i j} \widehat{p_{j}} \mid 1 \leq i \leq s\right\}, G:=\left\{\widehat{m_{j}}+\sum_{i=1}^{m} \lambda_{i j} \widehat{p_{i}} \mid 1 \leq j \leq m\right\}
$$

and $C:=\left\{\boldsymbol{x}^{c}\left|c \in \mathbb{N}^{n},\right| c \mid=\mathcal{N}+1\right\}$. Then $G \cup W \cup C$ is a Gröbner basis for $Q$ with respect to $\prec$.

Proof. Proof of Proposition 3.7 in [28] works here as well. We just need to note that for every $f \in Q, l t(f) \in\langle l t(G) \cup l t(W) \cup C\rangle$.

Note that unlike in Proposition 3.7 in [28] $G \cup C$ is not a Gröbner basis in this case as we don't necessarily have $\langle l t(Q)\rangle=\langle l t(G) \cup C\rangle$.

Example 2. Let $I=\left\langle f_{1}, f_{2}\right\rangle \unlhd \mathbb{K}[x, y]$, where $f_{1}=x-y+x^{2}$ and $f_{2}=x-y+y^{2}$. In step 2 of the algorithm we have that

$$
M_{2}=\begin{aligned}
& \text { Condition (3) } \\
& \Lambda\left(f_{1}\right)=0 \\
& \Lambda\left(f_{2}\right)=0
\end{aligned}\left(\begin{array}{cccc}
d_{1} & d_{2} & d_{1}^{2} & d_{1} d_{2}+d_{2}^{2} \\
1 & 0 & 1 & -1 \\
1 & -1 & 1 & 0 \\
1 & 0 & 1
\end{array}\right)
$$


from which we obtain $D_{2}=\left\{\Lambda_{1}=1, \Lambda_{2}=d_{1}+d_{2}, \Lambda_{3}=d_{2}+d_{1}^{2}+d_{1} d_{2}+d_{2}^{2}\right\}$. The active columns in $D_{2}$ are $v_{1}, v_{2}, v_{3}, v_{4}$, where $v_{i}$ refers to column $i$ and therefore matrix $M^{\prime}$ defined in (5) (ignoring $\Lambda_{1}=1$ ) is

$$
\begin{aligned}
& \begin{array}{llll}
d_{1} & d_{2} & d_{1}^{2} & d_{1} d_{2}+d_{2}^{2}
\end{array} \\
& M^{\prime}=\Lambda_{2} \Lambda_{3}\left(\begin{array}{llll}
1 & 1 & 0 & 0 \\
0 & 1 & 1 & 1
\end{array}\right) \text {. }
\end{aligned}
$$

Two instances of substituting some columns of $M^{\prime}$ and then computing its (reduced) echelon form are shown below. Matrix

$$
\begin{aligned}
& \begin{array}{llll}
d_{2} & d_{1}^{2} & d_{1} & d_{1} d_{2}+d_{2}^{2}
\end{array} \\
& G_{1}^{\prime-1} M^{\prime} P^{\prime}=\Lambda_{2}^{\prime}\left(\begin{array}{cccc}
1 & 0 & -1 & 0 \\
0 & 1 & 1 & 1
\end{array}\right)
\end{aligned}
$$

gives columns $v_{2}$ and $v_{3}$ and matrix

$$
G_{2}^{\prime-1} M^{\prime} P^{\prime}=\Lambda_{2}^{\prime}\left(\begin{array}{cccc}
d_{2} & d_{1} d_{2}+d_{2}^{2} & d_{1} & d_{1}^{2} \\
\Lambda_{3}^{\prime} & 0 & -1 & 0 \\
0 & 1 & 1 & 1
\end{array}\right)
$$

gives columns $v_{2}$ and $v_{4}$.

For instance, if we consider $G_{2}^{\prime-1} M^{\prime} P^{\prime}$, then $\Lambda_{2}^{\prime}=d_{2}+d_{1}+d_{1}^{2}$ and $\Lambda_{3}^{\prime}=$ $d_{1} d_{2}+d_{2}^{2}+d_{1}+d_{1}^{2}$. Since $d_{2}$ only appears in $\Lambda_{2}^{\prime}$ and $d_{1} d_{2}+d_{2}^{2}$ only appears in $\Lambda_{3}^{\prime}$, by deleting columns $v_{2}$ and $v_{4}$ from $M_{3}$ we will have the following matrix in step 3 .

$$
M_{3}^{\lambda_{2}, \lambda_{4}}=\begin{array}{cccc}
d_{1} & d_{1}^{2} & d_{1}^{3}-d_{1}^{2} & d_{2}^{3}+d_{1} d_{2}^{2}+d_{1}^{3} d_{2}-d_{1} d_{2} \\
\text { Condition (2) } \\
\text { Condition (3) } \\
\Lambda\left(f_{1}\right)=0 \\
\Lambda\left(f_{2}\right)=0
\end{array}\left(\begin{array}{cccc}
0 & 0 & 1 & 1 \\
0 & 1 & 0 & 0 \\
1 & 1 & -1 & 0 \\
1 & 0 & 0 & 0
\end{array}\right) .
$$

Since $\operatorname{Ker}\left(M_{3}^{\lambda_{2}, \lambda_{4}}\right)=0$, the algorithm terminates. Using any of the pairs of columns obtained via other possible matrices the same basis would be obtained.

Modifications of Macaulay's Algorithm. The same idea can be used to delete some columns from the matrices obtained via Macaulay's algorithm. In this case, equivalent versions of Proposition 1 and Corollary 2 can be stated with slight modifications. For details see [25, 29].

The necessary modifications to Proposition 1 in order to adjust it to Macaulay's algorithm, are due to the different labeling of the matrices in the integration method and Macaulay's algorithm. In Macaulay's matrices, a column being active is reduced to having its label in the support of the dual basis elements in the previous step. In order to detect the columns to be deleted, one could construct matrix $M^{\prime}$ in (5) with the labeling in Macaulay's matrices and change it into the echelon form. This will lead to the criterion for deleting active columns in Macaulay's algorithm, i.e., the modification of Corollary 2. 
One can use the idea behind the integration method, that the monomials of the dual basis elements of degree $t$ can be obtained by integrating only the elements of the dual basis of degree $t-1$, in order to find some monomials labeling some columns of Macaulay's matrices in step $t$ that cannot be obtained via integration and therefore cannot appear in the basis elements of degree $t$. This helps with deleting those columns in advance, which will reduce the size of the matrices in Macaulay's algorithm even further. After applying this trick, the monomials labeling the columns of the matrices in Macaulay's algorithm will all appear as monomials in the polynomials labeling the matrices of the integration method.

We explain the above improvement by redoing the calculations for step 3 of Example 1, using the above modifications on Macaulay's algorithm and comparing the computations.

Example 3. After doing the computations in step 2, we have $D_{2}=\left\{1, d_{1}, 2 d_{1}^{2}+\right.$ $\left.d_{2}\right\} . d_{2} \in \operatorname{supp}\left(D_{2}\right)$, but $d_{2}^{2} \notin \operatorname{supp}\left(D_{2}\right)$. So, in step 3, by the above improvement, we can remove $v_{d_{1} d_{2}^{2}}$ and $v_{d_{2}^{3}}$ from $M_{3}$. Also we can remove the columns $v_{1}, v_{d_{1}}, v_{d_{1}^{2}}$ using the modifications induced by Proposition 1. So the new matrix has 5 columns, while the original matrix in Macaulay's method has 10 columns.

Change of the Integration Order at Each Step. We conclude by another possible optimization strategy. One can change the order of the variables at each step of the integration method in order to gain some computational advantage. Suppose that we have computed $D_{t-1}=\left\{\Lambda_{1}, \ldots, \Lambda_{m}\right\}$. Consider $n_{i}:=\#\left\{d x_{i}^{\alpha_{i}} \in \bigcup_{i} \operatorname{supp}\left(\Lambda_{i}\right) \mid \alpha_{i} \in \mathbb{N}\right\}$. Now, re-order the variables in the following way: if $n_{i} \leq n_{j}$ then $x_{i}<x_{j}$. We call such an order a good integrable order. Assume that $x_{b_{1}}<x_{b_{2}}<\ldots<x_{b_{n}}$ is a good integrable order, where $b_{i} \in\{1, \ldots, n\}$. Now we consider $\Lambda_{1}, \ldots, \Lambda_{m}$ as polynomials in $\mathbb{K}\left[d x_{b_{1}}, \ldots, d x_{b_{n}}\right]$ and continue with the integration in the following order:

$$
\begin{aligned}
\Lambda= & \left.\sum_{i} \lambda_{i 1} \int_{b_{1}} \Lambda_{i}\right|_{d x_{b_{2}}=\cdots=d x_{b_{n}}=0} \\
& +\cdots \\
& +\left.\sum_{i} \lambda_{i n-1} \int_{b_{n-1}} \Lambda_{i}\right|_{d x_{b_{2}}=\cdots=d x_{b_{n}}=0} \\
& +\sum_{i} \lambda_{i n} \int_{b_{n}} \Lambda_{i} .
\end{aligned}
$$

This way, the number of integrations is minimized. Note that the number of integrands and the number of basis elements of $D_{t-1}$ are fixed and therefore there is no advantage in terms of the size of $M_{t}$. The following example illustrates this optimization.

Example 4. Consider Example 1. In step two we have that

$$
D_{2}=\left\langle\Lambda_{1}=1, \Lambda_{2}=d_{1}+d_{2}, \Lambda_{3}=-d_{1}+d_{1}^{2}+d_{1} d_{2}+d_{2}^{2}\right\rangle .
$$


Then $n_{1}=3, n_{2}=2$. Therefore we change the order into $y<x$ and work on $\mathbb{K}[d y, d x]$. Then

$\Lambda=\lambda_{1} d y+\lambda_{2} d x+\lambda_{3} d y^{2}+\lambda_{4}\left(d y d x+d x^{2}\right)+\lambda_{5}\left(d y^{3}\right)+\lambda_{6}\left(d x^{3}-d x^{2}+d x^{2} d y+d x d y^{2}\right)$.

There is only one monomial in the 5-th column of $M_{3}$, while in the original ordering, there were two:

$$
\begin{aligned}
\Lambda= & \lambda_{1} d x+\lambda_{2} d y+\lambda_{3} d x^{2}+\lambda_{4}\left(d x d y+d y^{2}\right) \\
& +\lambda_{5}\left(d x^{3}-d x^{2}\right)+\lambda_{6}\left(d y^{3}+d x d y^{2}+d x^{2} d y+d x d y\right) .
\end{aligned}
$$

\section{Directional Multiplicity}

In this section, using the dual space of an ideal, we introduce the notion of Directional Multiplicity. Directional multiplicity provides a lot of information about the multiplicity structure at an isolated point. Using Lemma 9 we give a sound definition of directional multiplicity. Then, we show that directional multiplicities can be bounded and can bound other invariants of an ideal, namely the Nil-index and the intersection multiplicity.

Definition and Properties. The first step towards a sound definition of directional multiplicity is to observe that the set of monomials that appear in elements of $Q^{\perp}$ is exactly the set of monomials $\partial^{\mathbf{a}}$ such that $x^{\mathbf{a}} \notin Q$, where $x^{\mathbf{a}}=x_{1}^{a_{1}} \cdots x_{n}^{a_{n}}$. Note that

$$
\partial^{\mathbf{a}}\left(x^{\mathbf{b}}\right)=\prod \delta_{a_{i}, b_{j}}
$$

where $\delta_{i, j}$ is the Kronecker delta. We prove the following result that first appeared without a proof in [23].

Proposition 7 (Characterization of Monomials in $Q^{\perp}$ ). Let $Q=Q_{\zeta}$ be an

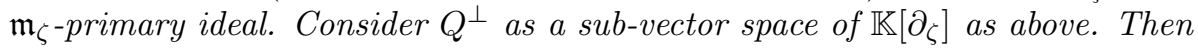

$$
\bigcup_{\Lambda \in Q^{\perp}} \operatorname{supp}(\Lambda)=\left\{\partial^{\boldsymbol{a}} \mid x^{\boldsymbol{a}} \notin Q\right\}
$$

where $\operatorname{supp}(\Lambda)$ is the set of monomials with nonzero coefficient in $\Lambda$.

Proof. By Theorem 3.2 in [28], we have that $f \in Q \Leftrightarrow\left(\lambda(f)=0\right.$ for all $\left.\lambda \in Q^{\perp}\right)$. This implies that $f \in Q \Leftrightarrow(\lambda(f)=0$ for all $\lambda \in D)$, for a basis $D \subset \mathbb{K}[\partial]$ of $Q^{\perp}$.

We are ready to prove the thesis:

"ᄃ" If $\partial^{\mathbf{a}}$ is in $\operatorname{supp}(\Lambda)$ then the monomial $x^{\mathbf{a}}$ is not annihilated by $\Lambda$ (see (7)), which implies $x^{\mathbf{a}} \notin Q$.

"Р" If $x^{\mathbf{a}} \notin Q$, then there exists $\lambda \in D$ such that $\lambda\left(x^{\mathbf{a}}\right) \neq 0$. Let $\Lambda \in \mathbb{K}[\partial]$ be the differential operator corresponding to $\lambda$, so $\Lambda\left(x^{\mathbf{a}}\right) \neq 0$. By (7), we know that $m\left(x^{\mathbf{a}}\right)=0$ for all monomials $m$ in $\operatorname{supp}(\Lambda)$ which are different from $\partial^{\mathbf{a}}$. Hence $\partial^{\mathbf{a}}$ has to be in $\operatorname{supp}(\Lambda)$. 
After characterizing the monomials in $Q^{\perp}$, the next step is to study their behaviour under projection. Let $I$ be an ideal in $R$ and $I_{2, \ldots, n}=I \cap \mathbb{K}\left[x_{2}, \ldots, x_{n}\right]$ be its first elimination ideal. The following result shows that the objects introduced so far, behave well in the framework of elimination theory.

Proposition 8 ([9], Proposition 7.19 ). Let $\pi$ be the linear map

$$
\begin{array}{cccc}
\pi: \mathbb{K}\left[\left[d x_{1}, \ldots, d x_{n}\right]\right] & \longrightarrow & \mathbb{K}\left[\left[d x_{2}, \ldots, d x_{n}\right]\right] \\
\Lambda & \mapsto & \Lambda\left(0, d x_{2}, \ldots, d x_{n}\right) .
\end{array}
$$

Then $I_{2, \ldots, n}^{\perp}=\pi\left(I^{\perp}\right)$.

We use the above proposition in order to prove the Dual Projection Lemma, which shows how to get a basis of the dual space of the elimination ideal, having a basis for the dual space. Note that $I$ in Proposition 8 can be any ideal, however the following lemma is stated only for the local case, i.e., when we are working on an $\mathfrak{m}_{\zeta}$-primary ideal $Q=Q_{\zeta}$.

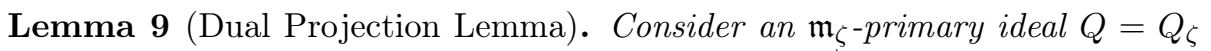
and let $D=\left\{\Lambda_{0}, \Lambda_{1}, \ldots, \Lambda_{\ell-1}\right\} \subset \mathbb{K}[\partial]$ be a basis of $Q^{\perp}$. Then

$$
Q_{2, \ldots, n}^{\perp}=\left\langle\left.\Lambda_{0}\right|_{d x_{1}=0},\left.\Lambda_{1}\right|_{d x_{1}=0}, \ldots,\left.\Lambda_{\ell-1}\right|_{d x_{1}=0}\right\rangle .
$$

Proof. The projected elements of $D$ are in $Q_{2, \ldots, n}^{\perp}$. They generate the dual space of the elimination ideal, since by Proposition 8 the projection $\pi$ from $Q^{\perp}$ is onto $Q_{2, \ldots, n}^{\perp}$.

The following immediate corollary deals with the case of projection to one variable.

Corollary 10. Let $D=\left\{\Lambda_{0}, \Lambda_{1}, \ldots, \Lambda_{l-1}\right\} \subset \mathbb{K}[\partial]$ be a basis of $Q^{\perp}$, and for $1 \leq i \leq n$ let $Q_{i}=Q \cap \mathbb{K}\left[x_{i}\right]$. Denote by $\left.\Lambda\right|_{d x_{i} \neq 0}$ the polynomial obtained by substituting $d x_{j}=0$ for $1 \leq i \neq j \leq n$ in $\Lambda$. Then

$$
Q_{i}^{\perp}=\left\langle\left.\Lambda_{0}\right|_{d x_{i} \neq 0},\left.\Lambda_{1}\right|_{d x_{i} \neq 0}, \ldots,\left.\Lambda_{l-1}\right|_{d x_{i} \neq 0}\right\rangle .
$$

Moreover, there exists $\mu_{i} \in \mathbb{N}$ such that

$$
Q_{i}^{\perp}=\left\langle 1, d x_{i}, \ldots, d x_{i}^{\mu_{i}-1}\right\rangle .
$$

Now we have the necessary tools to define the directional multiplicity.

Definition 1 (Directional Multiplicity). Let $\zeta$ be an isolated point in the variety of an ideal $I$ and $Q_{\zeta}$ be the corresponding $\mathfrak{m}_{\zeta}$-primary component. Using the notation of Corollary 10, for $1 \leq i \leq n$, we define the $i$-th directional multiplicity of $\zeta$ to be $\mu_{i}$ and denote it by $\mu_{i}(\zeta)$ or simply $\mu_{i}$ if $\zeta$ is clear from context. 


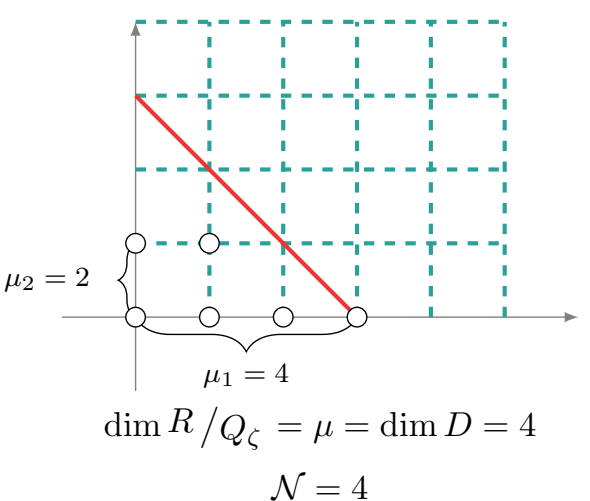

Figure 1: Extended Buchberger Diagram for Example 1

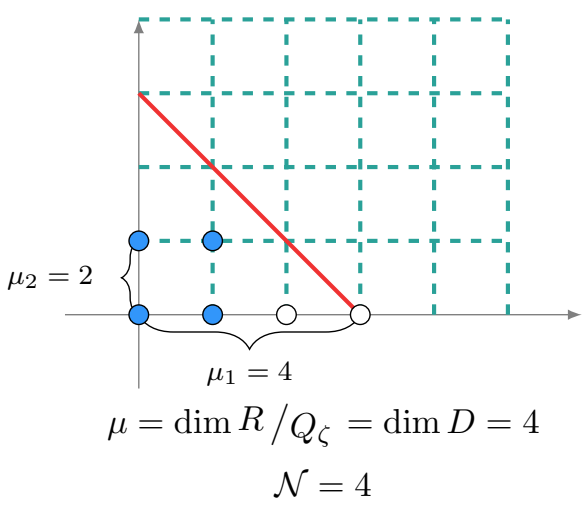

Figure 2: Extended Buchberger diagram vs a basis for $R / Q_{\zeta}$ with respect to a degree ordering

In order to give an intuition of directional multiplicity, we take a look at the quotient $R / Q_{\zeta}$. If we consider this quotient as a vector space, then a basis for this space is given by the monomials in the, so called, Buchberger diagram, which consists of all monomials which are not contained in the initial (i.e., leadingterm) ideal of the Gröbner basis [4]. Let us recall that the multiplicity of $\zeta$ is defined as $\operatorname{dim}_{\mathbb{K}} R / Q_{\zeta}$. We will denote the multiplicity by $\mu(\zeta)$ or simply by $\mu$ if $\zeta$ is clear from the context.

As discussed earlier, we can find the monomials of $D$ by searching among those monomials of $I^{\perp}$ that have degree at most the Nil-index. These monomials are actually the monomials under the Extended Buchberger Diagram which is defined below.

Definition 2 (Extended Buchberger Diagram). The Extended Buchberger Diagram of an $\mathfrak{m}_{\zeta}$-primary ideal $Q_{\zeta}$ is obtained by considering all the monomials that appear in a basis of the dual space of $Q_{\zeta}$.

We can think of the Nil-index of $Q_{\zeta}$ as the largest degree of the monomials under the extended Buchberger diagram. Figure 1 shows the extended Buchberger diagram and all of its monomials for Example 1.

Note that the monomials under the Buchberger diagram with respect to an ordering form a vector space basis for $R / Q$. They include some monomials in a basis of $Q^{\perp}$, but they do not necessarily include all the monomials in $D$. In particular, they may not include the highest powers of $d x_{i}$, i.e., the monomials corresponding to the directional multiplicities. However in the extended Buchberger diagram, one can see all the possible monomials in $D$, which are all the monomials that do not appear in $Q$. These monomials include all the monomials in the Buchberger diagram of $Q$.

The above comments are illustrated in Figure 2. The black dots show a basis for $R / Q$, while the white dots are the rest of the monomials in the basis of $Q^{\perp}$, see also [24] for a similar diagram. Also Figures 3 and 4 show the quotient of 


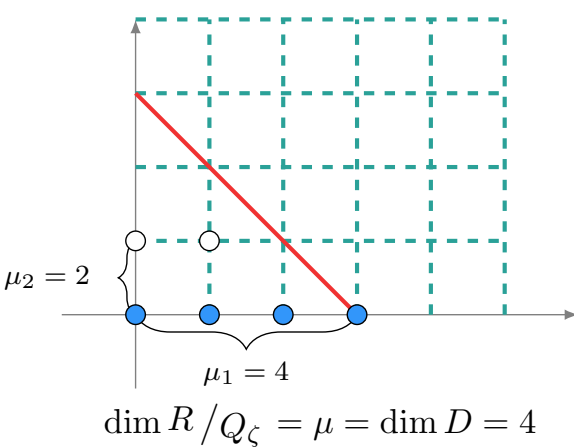

Figure 3: Extended Buchberger diagram vs directional multiplicity with respect to $x$ for Example 1

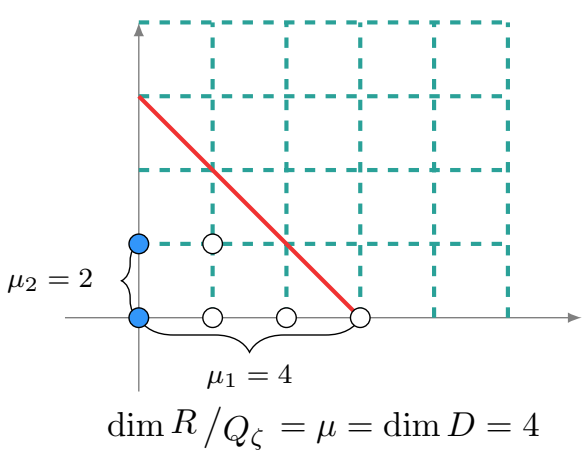

Figure 4: Extended Buchberger diagram vs directional multiplicity with respect to $y$ for Example 1

the elimination ideal with respect to $x$ and the quotient of the elimination ideal with respect to $y$, respectively. In Figure 3, black dots are the basis for $Q_{2}^{\perp}$ and the white dots are the rest of the monomials in the dual basis. In Figure 4, black dots are the basis for $Q_{1}^{\perp}$ and the white dots are the rest of the monomials in the dual basis.

Considering the above figures, one can see that the extended Buchberger diagram includes the Buchberger diagram with respect to every order. $\mathcal{N}$ is a bound for the degree of the members of a Gröbner basis with respect to every order. Directional multiplicity with respect to an axis is the largest intersection point of the extended Buchberger diagram with that axis. The Buchberger diagram does not necessarily have an intersection with the hyperplane $x_{1}+$ $\cdots+x_{n}=\mathcal{N}$, but the extended Buchberger diagram does have at least a point in common with that hyperplane.

Example 5. Let $I=\left\langle f_{1}=x^{8}+y^{5}, f_{2}=x^{7} y^{4}\right\rangle$. The origin is a root of the system with multiplicity $\mu=67$. We have that $\mathcal{N}=18$, while $\mu_{1}=15, \mu_{2}=9$. The reduced Gröbner basis for I with respect to the lexicographic order $(x>y)$ is $\left\{f_{1}=x^{8}+y^{5}, f_{2}=x^{7} y^{4}, g_{y}=y^{9}\right\}$, and with respect to lexicographic order $(y>x)$ is $\left\{f_{1}=y^{5}+x^{8}, f_{2}=y^{4} x^{7}, g_{x}=x^{15}\right\}$, where $g_{y}$ and $g_{x}$ are the generators of the elimination ideal with respect to the lexicographic orders $x>y$ and $y>x$ respectively.

These observations give us the intuition that the directional multiplicities are at most as large as the Nil-index. Also their product gives us the volume of a cuboid which contains the extended Buchberger diagram. The following statements make the comments above more precise.

Let us first note that the Nil-index is at most as large as the multiplicity and the multiplicity is bounded by the number of lattice points in the n-simplex. Thus $\mathcal{N} \leq \mu \leq$ Number of Lattice point in the n-simplex $=\left(\begin{array}{c}\mathcal{N}+n \\ n\end{array}\right)$.

Proposition 11. Let $\mu$ be the multiplicity of an isolated point $\zeta$. Then 
- $\mu_{i} \leq \mu \quad$ for every $1 \leq i \leq n$.

- $\mu \leq \prod_{1 \leq i \leq n} \mu_{i}$.

- $\sum_{i=1}^{n} \mu_{i}-n+1 \leq \mu$.

Proof. For the first part, recall that $\operatorname{dim}_{\mathbb{K}} Q_{\zeta}^{\perp}=\mu$ and that $\mu_{i}$ is the dimension of a vector subspace of $Q_{\zeta}^{\perp}$. Thus $\mu_{i} \leq \mu$.

For the second part, for every $1 \leq i \leq n, \mu_{i}$ is the largest degree of the elements in $Q_{\zeta}^{\perp} \cap \mathbb{K}\left[d_{i}\right]$. This means that $\mu_{i}+1$ is the largest possible degree of $x_{i}$ in $R / Q$. Since $\mu=\operatorname{dim}_{\mathbb{K}} R / Q$, we conclude that $\mu \leq \prod_{1 \leq i \leq n} \mu_{i}$.

For the third statement, note that as argued above, $d x_{i}^{a_{i}} \in Q_{\zeta}^{\perp}$ if and only if $a_{i}<\mu_{i}$. This means that $x_{i}^{a_{i}} \notin Q_{\zeta}$ if and only if $a_{i}<\mu_{i}$. Now, for all $1 \leq i \leq n$, let $A_{i}:=\left\{1, x_{i}, \cdots, x_{i}^{\mu_{i}-1}\right\}$. Then, $\left\langle\bigcup A_{i}\right\rangle \subseteq R / Q_{\zeta}$ as vector spaces. Note that the elements of $\bigcup A_{i}$ are linearly independent. Then $\operatorname{dim}\left\langle\bigcup A_{i}\right\rangle=$ $\sum \mu_{i}-n+1 \leq \operatorname{dim} R / Q_{\zeta}=\mu$ and the result follows.

Proposition 12. Let $\mathcal{N}$ be the Nil-index of $Q_{\zeta}$. Then

- $\mathcal{N} \geq \mu_{i}$ for all $1 \leq i \leq n$,

- $\mathcal{N} \leq \sum_{1 \leq i \leq n} \mu_{i}-n$

Proof. According to the definition of the Nil-index we have $\mathfrak{m}_{\zeta}^{\mathcal{N}} \nsubseteq Q_{\zeta}$ and $\mathfrak{m}_{\zeta}^{\mathcal{N}+1} \subseteq Q_{\zeta}$. Since $\mathfrak{m}_{\zeta}^{\mathcal{N}}=\left\langle x_{1}-\zeta_{1}, \ldots, x_{n}-\zeta_{n}\right\rangle^{\mathcal{N}}$, we have that $\left(x_{i}-\zeta_{i}\right)^{\mathcal{N}} \notin$ $Q_{\zeta}$ and $\left(x_{i}-\zeta_{i}\right)^{\mathcal{N}+1} \in Q_{\zeta}$. By the definition of $\mu_{i}$ and the Proposition 7, $d x_{i}^{\mu_{i}}\left(x_{i}-\zeta_{i}\right)^{\mathcal{N}}=0$ and $d x_{i}^{\mu_{i}}\left(x_{i}-\zeta_{i}\right)^{\mathcal{N}-1} \neq 0$. Therefore $\mu_{i} \leq \mathcal{N}$.

For the second part, note that for all $x_{i}, d_{x_{i}}^{\mu_{i}-1} \in \operatorname{supp}\left(Q_{\zeta}^{\perp}\right)$ and $d_{x_{i}}^{\mu_{i}} \notin$ $\operatorname{supp}\left(Q_{\zeta}^{\perp}\right)$. Therefore by Proposition $7, x_{i}^{\mu_{i}-1} \notin Q_{\zeta}$ and $x_{i}^{\mu_{i}} \in Q_{\zeta}$. Consider $A=\left\{\mathbf{a} \in \mathbb{N}^{n}|\quad| \mathbf{a} \mid=\sum\left(\mu_{i}-1\right)+1\right\}$. By the Pigeonhole principle, there exists an $i, 1 \leq i \leq n$, such that $x_{i}^{\mu_{i}} \mid x^{\mathbf{a}}$. Therefore $x^{\mathbf{a}} \in Q_{\zeta}$ for all $\mathbf{a} \in A$, which implies that $\mathfrak{m}_{\zeta}^{|\mathbf{a}|} \subseteq Q_{\zeta}$ and $\mathcal{N}<|\mathbf{a}|=1+\sum\left(\mu_{i}-1\right)$. The result follows by minimality of $\mathcal{N}$.

The inequalities in the Propositions 11 and 12 are sharp. For example, in the univariate case, where $I=Q_{\zeta} \in \mathbb{K}[x]$, we have $\mathcal{N}=\mu_{1}=\mu$.

A geometric interpretation of the $i$-th directional multiplicity at an intersection point is the number of instances of the intersection point that can be seen when we look at the intersection point in the direction parallel to the $x_{i}$ axis. Some of the presented inequalities are direct consequences of the definitions, and reveal interesting properties of this new notion. In particular, knowing the directional multiplicities we can deduce information about the multiplicity or the Nil-index. Thus, the notion of directional multiplicity is, in this sense, a refinement of multiplicity and Nil-index. Moreover, in applications this refined 
information can be used. In particular, methods such as the subdivision of [1], for the arrangement of several planar curves produces small boxes which are likely to contain isolated singularities. Taking the center of such a box as an approximation of a multiple root allows for an approximate dual computation that can reveal directions that lead to degenerate computations.

Geometry of the Elimination Ideal. Let $I \subseteq \mathbb{C}[x, y]$ be a zero dimensional ideal with no roots at infinity generated by two polynomials corresponding to two planar curves and $I_{1}=I \cap \mathbb{C}[y]=\langle g\rangle$ be its elimination ideal. We illustrate the case of geometric degeneracy and how directional multiplicity can be used, in a concrete example. Let $f_{1}=(y+1)(y-x+1)$ and $f_{2}=x^{2}+y^{2}-1$ as shown in the figure. The two curves intersect at two points, namely $(1,0)$ and $(0,-1)$. Their Sylvester resultant is $2 y(y+1)^{3}$, which implies that the projection on the $y$-axis of the roots $(1,0)$ and $(0,-1)$ have multiplicity 1 and 3 respectively. On the other hand, computing the Gröbner basis of the elimination ideal in $\mathbb{C}[y]$, we obtain the unique monic generator $g=y(y+1)^{2}$.

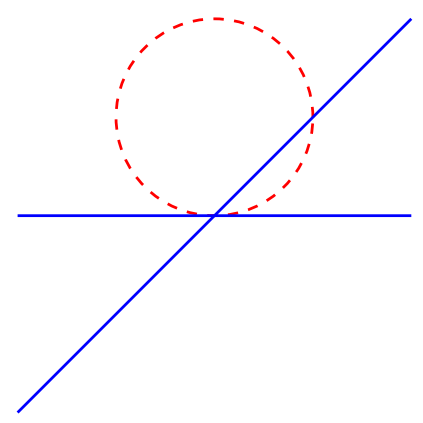

$$
\begin{array}{rl}
f_{1} & (y+1)(y-x+1) \\
f_{2} & x^{2}+y^{2}-1 \\
g & y(y+1)^{2} \\
\text { resultant } & 2 y(y+1)^{3}
\end{array}
$$

Observing the difference in the multiplicities of the resultant and $g$, the questions "when does the multiplicity drop?" and "what does the multiplicity of a factor in $g$ mean?" arise. Using the concept of directional multiplicity, we are able to address these questions in the degenerate case, as the one in the example.

The exponent of the factor of $g$ corresponding to an intersection point is the directional multiplicity at that point. The exponent of the corresponding factor of the resultant give us the multiplicity of the intersection points. However Gröbner basis did not say much about the geometry of the intersection. Now having the concept of directional multiplicity, we can explain the generator of the elimination ideal geometrically. In general given dense polynomials $f_{1}, \ldots, f_{n} \in$ $\mathbb{K}\left[x_{1}, \ldots, x_{n}\right]$, let $I_{1}=\langle g\rangle$ and $R_{1}, \ldots, R_{k}$ be the square-free factorization of the Macaulay resultant, with $R_{i}$ corresponding to the isolated point $\zeta_{i}$. Then $g=R_{1}^{\mu_{1}\left(\zeta_{1}\right)} \ldots R_{k}^{\mu_{1}\left(\zeta_{k}\right)}$.

\section{Benchmarks}

We compare the methods described in the previous sections with respect to both the size of the matrices produced and the running time. The methods we 
compare are the following:
MACAULAY
The Macaulay algorithm in [21]
INTEGMM
The integration method of [23]
INTEGMMO
The integration method of [23] with optimizations
NEWINTEG
The integration method with the criterion of Corrolary 2
NEWINTEGO
The integration method with the criterion of Corrolary 2 and optimizations

The polynomial systems we use for the benchmarks are the following:

\begin{tabular}{|c|c|c|}
\hline Name & Polynomials & Point \\
\hline cbms1 & $x^{3}-y z, y^{3}-x z, z^{3}-x y$ & $(0,0,0)$ \\
\hline cbms2 & $\begin{array}{l}x^{3}-3 x^{2} y+3 x y^{2}-y^{3}-z^{2}, z^{3}-3 z^{2} x+3 z x^{2}- \\
x^{3}-y^{2}, y^{3}-3 y^{2} z+3 y z^{2}-z^{3}-x^{2}\end{array}$ & $(0,0,0)$ \\
\hline mth191 & $x^{3}+y^{2}+z^{2}-1, x^{2}+y^{3}+z^{2}-1, x^{2}+y^{2}+z^{3}-1$ & $(0,1,0)$ \\
\hline decker2 & $x+y^{3}, x^{2} y-y^{4}$ & $(0,0)$ \\
\hline Ojika2 & $x^{2}+y+z-1, x+y^{2}+z-1, x+y+z^{2}-1$ & $(1,0,0)$ \\
\hline Ojika3 & $\begin{array}{l}x+y+z-1,2 x^{3}+5 y^{2}-10 z+5 z^{3}+5,2 x+2 y+ \\
z^{2}-1\end{array}$ & $(0,0,1)$ \\
\hline KSS & $\begin{array}{l}x^{2}-2 x+x+y+z+w+t-4, y^{2}-2 y+x+y+z+ \\
w+t-4, z^{2}-2 z+x+y+z+w+t-4, w^{2}-2 w+ \\
x+y+z+w+t-4, t^{2}-2 t+x+y+z+w+t-4\end{array}$ & $(1,1,1,1,1)$ \\
\hline Caprasse & $\begin{array}{l}-x_{1}{ }^{3} x_{3}+4 x_{1} x_{2}{ }^{2} x_{3}+4 x_{1}{ }^{2} x_{2} x_{4}+2 x_{2}{ }^{3} x_{4}+ \\
4 x_{1}{ }^{2}-10 x_{2}{ }^{2}+4 x_{1} x_{3}-10 x_{2} x_{4}+2,-x_{1} x_{3}{ }^{3}+ \\
4 x_{2} x_{3}{ }^{2} x_{4}+4 x_{1} x_{3} x_{4}{ }^{2}+2 x_{2} x_{4}{ }^{3}+4 x_{1} x_{3}+4 x_{3}{ }^{2}- \\
10 x_{2} x_{4}-10 x_{4}{ }^{2}+2, x_{2} x_{3}+2 x_{1} x_{2} x_{4}-2 x_{1}- \\
x_{3}, x_{1} x_{4}{ }^{2}+2 x_{2} x_{3} x_{4}-x_{1}-2 x_{3}\end{array}$ & $(2,-i \sqrt{(3)}, 2, i \sqrt{(3)})$ \\
\hline Cyclic 9 & $\begin{array}{l}\sum_{j=0}^{8} \prod_{k=j}^{i+j-1} x_{k} \bmod 9, \quad i=1, \ldots, 8 \quad \text { and } \\
1-x_{1} x_{2} x_{3} x_{4} x_{5} x_{6} x_{7} x_{8} x_{9}\end{array}$ & $\begin{array}{l}(-0.9383-0.3404 i \\
\ldots, 2.4602+0.89550 i)\end{array}$ \\
\hline DZ2 & $x^{4}, x^{2} y+y^{4}, z+z^{2}-7 x^{3}-8 x^{2}$ & $(0,0,-1)$ \\
\hline DZ3 & $\begin{array}{l}14 x+33 y-3 \sqrt{5}\left(x^{2}+4 x y+4 y^{2}+2\right)+\sqrt{7}+ \\
x^{3}+6 x^{2} y+12 x y^{2}+8 y^{3}, 41 x-18 y-\sqrt{5}+8 x^{3}- \\
12 x^{2} y+6 x y^{2}-y^{3}+3 \sqrt{7}\left(4 x y-4 x^{2}-y^{2}-2\right)\end{array}$ & $(1.5055,0.36528)$ \\
\hline hms $n$ & $x, y^{2}, z^{n}$ & $(0,0,0)$ \\
\hline
\end{tabular}

\subsection{Optimizations}

In the optimized versions of the algorithm we took care to improve the computing times. The size of the matrix mostly determines the computation times, therefore we optimize the algorithms by careful pivoting and by purging unnecessary columns and rows.

Pivoting. Below we show the role of pivoting within our improvement. Let us put an order on the monomials of $D_{t-1}$, e.g., degree lexicographic, and denote by $l t\left(\Lambda^{\prime}\right)$, the leading term of $\Lambda^{\prime}$, for every $\Lambda^{\prime} \in D_{t}$. Now one can reduce the members of a basis of $D_{t}$ with respect to each other, so that $l t\left(\Lambda^{\prime}\right) \notin$ $\operatorname{supp}\left(\Lambda^{\prime \prime}\right)$, for all $\Lambda^{\prime} \neq \Lambda^{\prime \prime} \in D_{t}$. We call such a basis a reduced basis. Then every leading term will be a monomial that uniquely appears in the reduced basis. If $\Lambda_{1}, \ldots, \Lambda_{m}$ is a basis for $D_{t-1}$, then removing the columns corresponding to $l t\left(\Lambda_{1}\right), \ldots, l t\left(\Lambda_{m}\right)$ from $M_{t}$ is equivalent to part 3 of Proposition 1. Using part 1 of Proposition 1, one can efficiently check whether $v_{\lambda}$ is active in $D$. This must 
be done with precise pivoting. For that, one must start with reducing $v_{\lambda}$ with the appropriate columns, without doing the column reductions for the other columns, unless it is required. As a side remark, using row echelon form is also taking advantage of pivoting.

Reduction of the number of rows in (3). Condition (3) expresses the fact that the dual is stable by derivation. The number of these polynomial conditions is $\left(\begin{array}{l}n \\ 2\end{array}\right)$, and each of them implies $m$ linear conditions on the coefficients. These conditions constitute the main part of the matrix. In practice we observed that many of these conditions are repeated for different combinations of $i$ and $k$ in (3), or vanish. Therefore, in the optimized versions of the algorithm we detect and purge repeating conditions before inserting them to the matrix.

Reduction of the number of rows and columns in (4). As already noted after Corollary 2, it can happen that some conditions in (4) have the form $\Lambda\left(x^{\beta_{i}}\right)=$ $\lambda_{i k}$. In this case a row appears in the matrix with a single non-zero element. Therefore both the row and the column $\lambda_{i k}$ can be removed, since they refer to a dual element which is already in our basis.

\subsection{Comparing Matrix Sizes}

As size is the biggest obstacle in computations, an important metric for the comparison of the different methods is the size of the matrices constructed. The following table presents the sizes of the matrices at step $t$ for each method, where $e$ is the number of the polynomials in the given basis, $n$ is the number of variables in the polynomial ring and $m$ is the dimension of $Q_{t-1}^{\perp}$ :

\begin{tabular}{|lll|}
\hline method & rows & columns \\
\hline MACAULAY & $\left(\begin{array}{l}t+n-1 \\
n\end{array}\right) e$ & $\left(\begin{array}{c}t+n \\
n\end{array}\right)$ \\
INTEGMM & $\left(\begin{array}{l}n \\
2\end{array}\right) m+e+m$ & $n m$ \\
NEWINTEG & $\left(\begin{array}{l}n \\
2\end{array}\right) m+e$ & $(n-1) m+1$ \\
\hline
\end{tabular}

Using our implementation and applying the optimizations discussed above, at the final step of the algorithms, we obtain matrices of the following sizes: 


\begin{tabular}{|l|ccccc|}
\hline & MACAULAY & INTEGMM & INTEGMMO & NEWINTEG & NEWINTEGO \\
\hline cbms1 & $105 \times 56$ & $46 \times 33$ & $27 \times 23$ & $36 \times 23$ & $27 \times 23$ \\
cbms2 & $60 \times 35$ & $34 \times 24$ & $21 \times 17$ & $27 \times 17$ & $21 \times 17$ \\
mth191 & $30 \times 20$ & $18 \times 12$ & $10 \times 9$ & $15 \times 9$ & $10 \times 9$ \\
decker2 & $20 \times 15$ & $9 \times 8$ & $5 \times 5$ & $6 \times 5$ & $5 \times 5$ \\
Ojika2 & $12 \times 10$ & $10 \times 6$ & $6 \times 5$ & $9 \times 5$ & $6 \times 5$ \\
Ojika3 & $60 \times 35$ & $18 \times 12$ & $12 \times 9$ & $15 \times 9$ & $12 \times 9$ \\
KSS & $630 \times 252$ & $180 \times 80$ & $155 \times 65$ & $165 \times 65$ & $155 \times 65$ \\
Caprasse & $60 \times 35$ & $31 \times 16$ & $22 \times 13$ & $28 \times 13$ & $22 \times 13$ \\
DZ2 & $360 \times 165$ & $66 \times 48$ & $44 \times 33$ & $51 \times 33$ & $44 \times 33$ \\
DZ3 & $30 \times 21$ & $11 \times 10$ & $6 \times 6$ & $7 \times 6$ & $6 \times 6$ \\
hms5 & $168 \times 84$ & $42 \times 30$ & $25 \times 21$ & $33 \times 21$ & $25 \times 21$ \\
hms7 & $360 \times 165$ & $58 \times 42$ & $35 \times 29$ & $45 \times 29$ & $35 \times 29$ \\
hms9 & $660 \times 286$ & $74 \times 54$ & $45 \times 37$ & $57 \times 37$ & $45 \times 37$ \\
hms11 & $1092 \times 455$ & $90 \times 66$ & $55 \times 45$ & $69 \times 45$ & $55 \times 45$ \\
hms20 & NA & $162 \times 120$ & $100 \times 81$ & $123 \times 81$ & $100 \times 81$ \\
hms30 & NA & $242 \times 180$ & $150 \times 121$ & $183 \times 121$ & $150 \times 121$ \\
cyclic9 & $495 \times 220$ & $156 \times 36$ & $114 \times 33$ & $153 \times 33$ & $114 \times 33$ \\
\hline
\end{tabular}

We observe that applying the optimizations described above in INTEGMMO, the extra rows added in INTEGMM are removed and the matrices obtained have exactly the same size as the ones obtained by the optimized version of the new method. Nevertheless, the non-optimized versions of the two methods produce matrices of different sizes, with the new method producing smaller ones. This has a direct effect in the time needed for the optimizations (as seen in the time comparisons).

We can observe that overall the optimization steps for INTEGMM and NEWINTEG have the same effect on the size of the matrices. The optimization on (3) applies to both INTEGMM and NEWINTEG, therefore, as expected, both methods are positively affected. The optimization on (4) refers to INTEGMM only. It should be noted, however, that this effect depends on the monomial ordering used to identify leading terms (we used a reverse total degree ordering). Different choice of the leading coefficients will not allow for this optimization. Interestingly, NEWINTEG does not depend on a choice of leading terms. A minimal number of columns is guaranteed by construction. However, it seems that the specific choice of ordering in INTEGMM also results in optimal matrices, after optimizations, as shown in the experiments.

\subsection{Comparing Running Times}

The benchmarks are performed in MAPLE 18 with 100 digits of precision. The hardware used is an 8 Intel $(R)$ Xeon $(R)$ CPU E5460 @ $3.1 \mathrm{GHz}$ core server with 16Gb or RAM, running Linux 3.16.0-4.

In the following table we provide the running times for computing the complete dual basis with the 5 different methods: 


\begin{tabular}{|l|ccccc|}
\hline & MACAULAY & INTEGMM & INTEGMMO & NEWINTEG & NEWINTEGO \\
\hline cbms1 & 2.256 & 0.335 & 0.240 & 0.256 & 0.219 \\
cbms2 & 0.812 & 0.171 & 0.127 & 0.139 & 0.132 \\
math191 & 0.259 & 0.064 & 0.056 & 0.060 & 0.056 \\
decker2 & 0.163 & 0.055 & 0.051 & 0.051 & 0.048 \\
Ojika2 & 0.055 & 0.044 & 0.035 & 0.036 & 0.036 \\
Ojika3 & 0.648 & 0.100 & 0.088 & 0.088 & 0.083 \\
KSS & 63.564 & 5.304 & 4.035 & 3.963 & 3.472 \\
Caprasse & 0.956 & 0.535 & 0.216 & 0.212 & 0.127 \\
DZ2 & 26.644 & 0.964 & 0.639 & 0.696 & 0.655 \\
DZ3 & 0.360 & 0.096 & 0.083 & 0.075 & 0.083 \\
hms5 & 4.808 & 0.284 & 0.199 & 0.215 & 0.207 \\
hms7 & 24.868 & 0.648 & 0.376 & 0.431 & 0.391 \\
hms9 & 108.088 & 1.156 & 0.683 & 0.803 & 0.708 \\
hms11 & 458.191 & 2.016 & 1.104 & 1.255 & 1.112 \\
hms20 & NA & 12.444 & 5.212 & 6.272 & 5.328 \\
hms30 & NA & 49.724 & 18.088 & 22.516 & 18.388 \\
cyclic9 & 32.247 & 5.012 & 5.028 & 4.860 & 3.883 \\
\hline
\end{tabular}

As expected, the methods producing the smaller matrices are faster, since the kernel computation at the end of each step is the bottleneck of all algorithms tested. In a few cases, timings are noticeably lower for NEWINTEGO than for INTEGMmo. The reason might be that less symbolic operations are performed in the new method, which can have impact when the system at hand is large and complicated, such as the cyclic9.

\section{Conclusions}

There are two main algorithms for computing the orthogonal of an $\mathfrak{m}_{\zeta}$-primary component of a given ideal. The first algorithm was given by Macaulay. Although it is still in use, and in spite of the improvements it saw since its inception, it is inefficient because of the construction of large matrices. The second algorithm, namely the integration method, reduces the size of the matrices drastically. In [23], the authors found a way to avoid redundant computations by computing a primal base for the quotient of the $\mathfrak{m}_{\zeta}$-primary ideal along with its dual base. This condition adds new rows to the matrices, which in some cases may lead to deleting those rows and some columns.

Our work generalizes the method presented in [23] in two ways. On one hand by introducing a criterion to avoid redundant computations, on the other hand by computing a general basis. This is done via computing a general basis for the quotient, which does not necessarily consist of monomials. Our method leads to deleting some columns from the matrices in the integration method instead of adding new rows. As shown in the benchmarks, the method produces consistently the smallest matrix among all methods tested. Moreover, in 
terms of practical performance, the benchmarks show that our implementation is consistently among the fastest of the methods tested.

Another aspect studied in the present work is that of local properties and geometry of an isolated point via its directional multiplicity. This gives the multiplicity structure with respect to an axis, and is useful in understanding the geometry behind projection. Moreover, it sheds light on the gap between the degree of the generator of the elimination ideal and the corresponding factor in the resultant.

Several research directions stem from the present work. A natural generalization would be to define the multiplicity in the direction of an arbitrary vector $v \in \mathbb{R}^{n}$, instead of only for the axis aligned directions. The directional multiplicities along these vectors can be used in studying singularities of curves. A second interesting aspect, arising naturally in almost all topics in elimination theory is sparsity. Exploiting the sparsity and structure of the matrices involved would improve the algorithms considerably. Last but not least, devising early termination criteria would be essential for the efficient computation of directional multiplicity.

\section{Acknowledgments}

The authors would like to express their sincere gratitude to Matteo Gallet for discussions and comments on the early versions of this work. The first author is grateful to his thesis advisor, B. Mourrain for introducing him to dual bases of singular isolated points. Part of this work has been done during the second author's visit to the Centre de Recerca Matemàtica in Bellaterra, Spain. The third author was partially supported by the project 113F293 under the program 1001 of the Scientific and Technological Research Council of Turkey. The authors are grateful to the reviewers for the useful remarks.

\section{References}

[1] L. Alberti, B. Mourrain, and J. Wintz. Topology and arrangement computation of semi-algebraic planar curves. Computer Aided Geometric Design, 25(8):631-651, 2008.

[2] J. G. Alcázar and G. M. Díaz-Toca. Topology of 2d and 3d rational curves. Computer Aided Geometric Design, 27(7):483 - 502, 2010.

[3] D. Bates, C. Peterson, and A. J. Sommese. A numerical-symbolic algorithm for computing the multiplicity of a component of an algebraic set. Journal of Complexity, 22(4):475-489, 2006.

[4] B. Buchberger. Ein Algorithmus zum Auffinden der Basiselemente des Restklassenringes nach einem Nulldimensionalen Polynomiideal (An Algorithm for Finding the Basis Elements in the Residue Class Ring Modulo a Zero Dimensional Polynomial Ideal). PhD thesis, Mathematical Institute, University of Innsbruck, Austria, 1965. (English translation in Journal of 
Symbolic Computation, Special Issue on Logic, Mathematics, and Computer Science: Interactions. Vol. 41, Number 3-4, Pages 475-511, 2006).

[5] B. Buchberger. Ein Algorithmisches Kriterium für die Lösbarkeit eines Algebraischen Gleichungssystems (An Algorithmic Criterion for the Solvability of Algebraic Systems of Equations) . Aequationes Mathematicae, 3:374-383, 1970. (English translation in B. Buchberger, F. Winkler (eds.): Gröbner Bases and Applications, London Math. Society Lecture Note Series 251, Cambridge Univ. Press, 1998, Pages 535 -545).

[6] R. M. Corless, G. M. Diaz-Toca, M. Fioravanti, L. Gonzalez-Vega, I. F. Rua, and A. Shakoori. Computing the topology of a real algebraic plane curve whose defining equations are available only "by values". Computer Aided Geometric Design, 30(7):675 - 706, 2013.

[7] B. H. Dayton and Z. Zeng. Computing the multiplicity structure in solving polynomial systems. In Proceedings of the 2005 International Symposium on Symbolic and Algebraic Computation, ISSAC '05, pages 116-123, New York, NY, USA, 2005. ACM.

[8] G. M. Diaz-Toca, M. Fioravanti, L. Gonzalez-Vega, and A. Shakoori. Using implicit equations of parametric curves and surfaces without computing them: Polynomial algebra by values. Computer Aided Geometric Design, 30(1):116 - 139, 2013. Recent Advances in Applied Geometry.

[9] M. Elkadi and B. Mourrain. Introduction à la Résolution des Systèmes Polynomiaux, volume 59. Springer, 2007.

[10] I. Emiris and B. Mourrain. Matrices in elimination theory. Journal of Symbolic Computation, 28(1-2):3-44, 1999.

[11] I. M. Gelfand, M. M. Kapranov, and A. Zelevinski. Discriminants, Resultants, and Multidimensional Determinants. Birkhäuser, 1994.

[12] J. D. Hauenstein, B. Mourrain, and A. Szanto. Certifying isolated singular points and their multiplicity structure. In Proceedings of the 2015 ACM on International Symposium on Symbolic and Algebraic Computation, ISSAC '15, pages 213-220, New York, NY, USA, 2015. ACM.

[13] Y. Huang and D. Wang. Computing intersection and self-intersection loci of parametrized surfaces using regular systems and Gröbner bases. Computer Aided Geometric Design, 28(9):566 - 581, 2011.

[14] R. Krone. Numerical algorithms for dual bases of positive-dimensional ideals. Journal of Algebra and Its Applications, 12(06):1350018, 2013.

[15] R. Krone and A. Leykin. Eliminating dual spaces. arXiv preprint arXiv:1503.02038, 2015. 
[16] A. Leykin, J. Verschelde, and A. Zhao. Higher-order deflation for polynomial systems with isolated singular solutions. In Algorithms in algebraic geometry, pages 79-97. Springer, 2008.

[17] N. Li and L. Zhi. Computing isolated singular solutions of polynomial systems: case of breadth one. SIAM Journal on Numerical Analysis, 50(1):354-372, 2012.

[18] N. Li and L. Zhi. Computing the multiplicity structure of an isolated singular solution: Case of breadth one. Journal of Symbolic Computation, 47(6):700 - 710, 2012.

[19] N. Li and L. Zhi. Verified error bounds for isolated singular solutions of polynomial systems. SIAM Journal on Numerical Analysis, 52(4):16231640, 2014.

[20] Z. Li and L. Zhi. Computing the nearest singular univariate polynomials with given root multiplicities. Theoretical Computer Science, 479:150 $162,2013$.

[21] F. S. Macaulay. The Algebraic Theory of Modular Systems. Cambridge mathematical library. Cambridge University Press, Cambridge, New York, Melbourne, 1994.

[22] T. Maekawa and N. M. Patrikalakis. Computation of singularities and intersections of offsets of planar curves. Computer Aided Geometric Design, 10(5):407 - 429, 1993.

[23] A. Mantzaflaris and B. Mourrain. Deflation and certified isolation of singular zeros of polynomial systems. In Proceedings of the 36th International Symposium on Symbolic and Algebraic Computation, ISSAC '11, pages 249256, New York, NY, USA, 2011. ACM.

[24] A. Mantzaflaris and B. Mourrain. Singular zeros of polynomial systems. In T. Dokken and G. Muntingh, editors, SAGA - Advances in ShApes, Geometry, and Algebra, volume 10 of Geometry and Computing, pages 77103. Springer International Publishing, 2014.

[25] A. Mantzaflaris, H. Rahkooy, and Z. Zafeirakopoulos. On directional multiplicities of isolated points. Technical Report 15-08, RISC, Hagenberg, Austria, 2015.

[26] M. Marinari, H. Möller, and T. Mora. On multiplicities in polynomial system solving. Transactions of the American Mathematical Society, 348(8):3283-3321, 1996.

[27] M. G. Marinari, T. Mora, and H. M. Möller. GrÖbner duality and multiplicities in polynomial system solving. In Proceedings of the 1995 International Symposium on Symbolic and Algebraic Computation, ISSAC '95, pages 167-179, New York, NY, USA, 1995. ACM. 
[28] B. Mourrain. Isolated points, duality and residues. Journal of Pure and Applied Algebra, 117:469-493, 1997.

[29] H. Rahkooy. Dual Space Algorithms for Computing Multiplicity Structure of Isolated Points. PhD thesis, RISC, Hagenberg, Austria, 2015.

[30] H. Rahkooy and Z. Zafeirakopoulos. Using resultants for inductive Gröbner bases computation. ACM Communications in Computer Algebra, 45(1), 2011.

[31] H. J. Stetter. Numerical Polynomial Algebra. SIAM, 2004.

[32] B. L. van der Waerden. Algebra, volume 1. Springer, New York, 7th edition, 1991. Based in part on lectures by E. Artin and E. Noether.

[33] B. L. van der Waerden. Algebra, volume 2. Springer, New York, 5th edition, 1991. Based in part on lectures by E. Artin and E. Noether.

[34] X. Wu and L. Zhi. Computing the multiplicity structure from geometric involutive form. In Proceedings of the Twenty-first International Symposium on Symbolic and Algebraic Computation, ISSAC '08, pages 325-332, New York, NY, USA, 2008. ACM.

[35] Z. Zeng. The closedness subspace method for computing the multiplicity structure of a polynomial system. Contemporary Mathematics, 496:347, 2009 . 